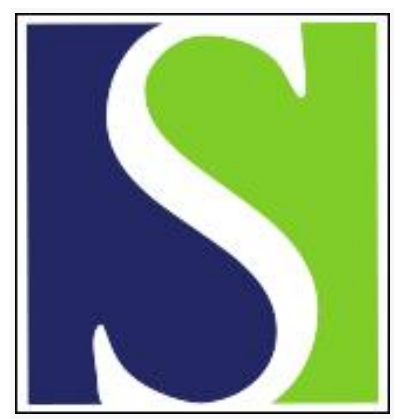

Scand J Work Environ Health 2004;30(2):85-128

https://doi.org/10.5271/sjweh.769

Issue date: Apr 2004

Is job strain a major source of cardiovascular disease risk?

by Belkic K, Landsbergis PA, Schnall PL, Baker D

Affiliation: Karolinska Institute, Department of Oncology-Medical Radiation Physics, PO Box 260, Stockholm, SE-17176 Sweden. Karen.Belkic@radfys.ki.se

Refers to the following texts of the Journal: 1996;22(4):241-242 1999;25(2):85-99 1997;23(4):257-265 1998;24(3):197-205 1996;22(2):139-145 1998;24(1):54-61 1998;24(5):334-343

The following articles refer to this text: 2004;30(2):81-83; 2006;32(6):431-442; 2006;32(6):473-481; 2006;32(6):515-527; 2007;33(1):1-3; 2008;34(1):48-54; 2008;34(1):40-47; SJWEH Supplements 2008;(6):33-40; SJWEH Supplements 2008;(6):41-51; 2008;34(4):288-296; 2009;35(4):284-293; 2011;37(6):455-463; 2012;38(6):489-502; 2013;39(1):106-111; 2014;40(5):441-456; 2015;41(3):280-287

Key terms: angina pectoris; cardiovascular death; cardiovascular disease; coronary artery disease; decision latitude; IHD; ischemic heart disease; job control; job strain; myocardial infarction; psychological demand; review; risk; skill discretion; work control

This article in PubMed: www.ncbi.nlm.nih.gov/pubmed/15127782

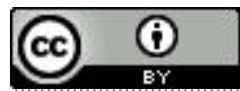




\title{
Is job strain a major source of cardiovascular disease risk? ${ }^{1}$
}

\author{
by Karen L Belkic, MD, ${ }^{2,3}$ Paul A Landsbergis, PhD, ${ }^{4}$ Peter L Schnall, MD, ${ }^{5}$ Dean Baker, MD ${ }^{5}$
}

\author{
Belkic K, Landsbergis PA, Schnall PL, Baker D. Is job strain a major source of cardiovascular disease risk? Scand \\ J Work Environ Health 2004;30(2):85-128.
}

\begin{abstract}
Empirical studies on job strain and cardiovascular disease (CVD), their internal validity, and the likely direction of biases were examined. The 17 longitudinal studies had the highest validity ratings. In all but two, biases towards the null dominated. Eight, including several of the largest, showed significant positive results; three had positive, nonsignificant findings. Six of nine case-control studies had significant positive findings; recall bias leading to overestimation appears to be fairly minimal. Four of eight cross-sectional studies had significant positive results. Men showed strong, consistent evidence of an association between exposure to job strain and CVD. The data of the women were more sparse and less consistent, but, as for the men, most of the studies probably underestimated existing effects. Other elements of causal inference, particularly biological plausibility, corroborated that job strain is a major CVD risk factor. Additional intervention studies are needed to examine the impact of ameliorating job strain upon CVD-related outcomes.
\end{abstract}

Key terms angina pectoris, cardiovascular death, coronary artery disease, decision latitude, ischemic heart disease, job control, myocardial infarction, psychological demands, skill discretion, work control.

Clinicians are often called upon to assess the cardiovascular work fitness of patients. As they attempt to make an informed judgment, a fundamental question arises: is the work environment fit, or conducive, to cardiovascular health? With technological advances, jobs characterized purely by heavy physical demands have become progressively less common. New types of workrelated challenges and burdens primarily affecting the higher nervous system of humans (ie, psychosocial stressors) are more and more frequently encountered. Yet most of the clinical guidelines relevant to the interface between the workplace and the patient's cardiovascular system continue to focus upon levels of physical exertion.

$\mathrm{Hu} \&$ Speizer (1) underscored the importance of identifying job-related and other environmental hazards that contribute to a given disease process. They noted that "physicians commonly treat the sequelae of such disease in the practice of medicine; however, unless the underlying connection with hazardous exposures is identified and mitigated, treatment of the manifestations rather than the cause at best only ameliorates the condition. At worst, the neglect of hazardous exposures may lead to both failure of treatment and failure to recognize a public health problem with wide significance [p 19]."

Several decades ago, occupational and environmental health research raised the concern that exposure to psychosocial stressors in the modern work environment may be related to cardiovascular disease (CVD). It was clear, however, that the evidence would be difficult to obtain, that a myriad of thorny methodological problems would arise, and that the critical obstacle would be the theoretical conceptualization, modeling, and measurement of workplace stressors. A major breakthrough came in 1979 with the introduction of the job strain (demand-control) model (2). The model was developed for work environments in which stressors are "chronic, not initially life-threatening and the product of sophisticated human organizational decision making. In decision

1 Portions of this paper were presented in abstracts to the 3rd International Congress on the Work Environment and Cardiovascular Disease, International Commission on Occupational Health, March 2002, Düsseldorf, Germany, and to the APA NIOSH Congress: Work, Stress and Health, March 2003, Toronto, Canada.

2 Karolinska Institute, Stockholm, Sweden.

3 Institute for Health Promotion and Disease Prevention Research, University of Southern California School of Medicine, Los Angeles, California, United States.

4 Department of Community Medicine, Mount Sinai School of Medicine, New York, New York, United States.

5 Division of Occupational \& Environmental Medicine, Department of Medicine, University of California at Irvine, College of Medicine, Irvine, California, United States.

Reprint requests to: Dr Karen Belkic, Adjunct Associate Professor of Preventive Medicine, Karolinska Institute, Department of Oncology-Medical Radiation Physics, PO Box 260, Stockholm, SE-17176 Sweden. [Karen.Belkic@ radfys.ki.se] 
making the controllability of the stressor is critical, and it becomes more important as increasingly complex and integrated social organizations develop, with ever more complex limitations on individual behavior [p 78]". The model has two components: "psychological demands, and a combined measure of task control and skill use, or decision latitude [p 78]". Job strain occurs when the human organism is overloaded psychologically and at the same time deprived of control over the work environment, a combination which is predicted to give rise to increased risk of stress-related illness (3, p 78). The basic components of the two dimensions are summarized in table 1. A third dimension, social isolation, was later added to the model, with the worst situation being "iso-strain": high demands, low decision-making latitude, plus lack of social support (5).

Exposure to job strain can be assessed from self-report via a questionnaire, with the dimensions operationalized in the form of short, general instruments, most frequently the job content questionnaire (JCQ) or the psychosocial job strain questionnaire (PSJSQ) (6-8). These measures are feasibly administered in field and epidemiologic studies. Data linkage methods have been developed in the United States and Sweden, so that exposure to job strain (as well as to "iso-strain" in Sweden) can also be inferred from occupational titles alone [ie, the imputation method (9-10)]. External assessment of job characteristics (eg, expert observer) is yet another method for obtaining exposure data. [See the work of Greiner \& Krause (11).]

The job-strain model has been the model most widely used for evaluating the psychosocial work environment and its potential impact upon the cardiovascular system,

Table 1. Basic components of the job-strain model, derived from the work of Karasek \& Theorell (4).

\begin{tabular}{|c|c|}
\hline Component & Demand \\
\hline Psychological job demands & $\begin{array}{l}\text { Job requires very hard work } \\
\text { Job requires very fast work } \\
\text { Job requires excessive work } \\
\text { Job involves conflicting demands } \\
\text { Job involves not having enough time to get } \\
\text { the job done }\end{array}$ \\
\hline Decision latitude & \\
\hline Skill discretion & $\begin{array}{l}\text { Job requires learning new things } \\
\text { Job provides opportunities to develop one's } \\
\text { skills } \\
\text { Job requires a high level of skill } \\
\text { Job requires creativity } \\
\text { Job entails a variety of things to do } \\
\text { Job does not involve a lot of repetitive work }\end{array}$ \\
\hline Decision authority & $\begin{array}{l}\text { Job allows making one's own decision } \\
\text { Job provides a lot of freedom as to how the } \\
\text { work gets done } \\
\text { Job provides a lot of say on the job } \\
\text { Job allows taking part in decisions affecting } \\
\text { oneself }\end{array}$ \\
\hline
\end{tabular}

with some studies incorporating the third dimension of social isolation as well. Since the introduction of the model, many empirical investigations have been published concerning the relation between job strain and CVD outcomes, including acute myocardial infarction (MI), coronary artery disease (CAD), and CVD-related mortality. Many of these studies report significant positive findings, and job strain is increasingly receiving attention as a potential contributor to CVD (12-14). On the other hand, there have been several nonconfirmatory findings concerning job strain and CVD outcomes published in largescale studies. These results spurred some questions concerning the strength and consistency of the evidence.

Several in-depth reviews $(13,15-19)$ have been published concerning the empirical data on the etiologic role of psychosocial workplace factors (in some of these also outside work) and CVD. One of these reviews (16) focused explicitly on job strain. However, a comprehensive and systematic assessment of the internal validity of the current body of reported results is needed. A key issue yet to be addressed is the direction in which methodological issues would most likely be acting, delineating situations that would increase the likelihood of obtaining null results and those that could lead to an overestimation of any association.

In this paper, we have used a predefined set of criteria to examine each of the empirical investigations on job strain and CVD. The criteria were developed specifically to assess the methodological issues affecting the internal validity of studies on this topic, and, whenever possible, to identify the direction in which the results would most likely be affected. We have not only addressed the strength and consistency of the association, but also the other major elements of causal inference as well. Particular attention has been devoted to exploring the viability of alternative hypotheses, as well as the question of biological plausibility (ie, what are the possible mechanisms through which job strain could affect the cardiovascular system). This critical review has thereby sought to provide a more definitive answer to the question "Is job strain a major CVD risk factor?" The clinical implications of the conclusion have also been explored.

\section{Methods used for the empirical review}

\section{Identification and inclusion of relevant studies}

\section{Search strategy}

A computer-based search was carried out using Medline, via OVID, from 1966 to January 2002. The search terms were entered as text words in the title, abstract, or other searchable fields (mesh terms, etc). For the independent variable, the search terms were job strain, iso-strain, decision latitude, psychological demands, work control, 
job control, skill discretion, decision-making authority and intellectual discretion. These terms were combined with search terms for the dependent variable: myocardial infarction, angina pectoris, ischemic heart disease, coronary artery disease, and cardiovascular death. A senior medical information specialist replicated this strategy. Bibliographies of relevant articles and personal files were also reviewed.

\section{Inclusion criteria and procedure}

Articles were chosen for review if they fulfilled the following criteria: (i) exposure to job strain was assessed or imputed via its two major workplace dimensions, psychological demands plus any of the following: decision latitude, skill discretion, decision authority and decision control, as these directly relate to the dependent variable, (ii) any of the following were included as the dependent variable: CAD, manifestations of ischemic heart disease (IHD) (angina pectoris, MI) or mortality from cardiovascular causes, (iii) a case-control, cross-sectional or cohort design was used, (iv) the study was empirical, and (v) the complete study was published in English as a full-length article in a peer-reviewed journal. More than one publication by a given author or authors was included in the review insofar as either the group(s) under study, the endpoints, or the design differed. If two or more studies by the same author(s) offered complementary information but had the same design, endpoint, and study group, they were combined and analyzed together.

Whenever self-report tools other than the JCQ or PSJSQ $(7,20)$ or their earlier versions were used to assess the demand and control dimensions, two of the authors independently reviewed the described methods to determine whether they were sufficiently compatible with the job-strain model. This procedure was performed in a blinded fashion. Formulations focusing primarily on the individual's subjective reaction to the work environment (eg, "how stressed are you by ...") rather than on its objective characteristics were excluded. As a minimal guideline, it was required that at least one item from each of the major job-strain dimensions be included and that these queries be phrased identically to the original questionnaires or so closely as to be a measure of the original concept (eg, "hectic work" as a measure of psychological job demands). Insofar as the two reviewers disagreed, a third author served as an arbiter.

\section{Included studies}

A total of 35 articles was identified that met all the inclusion criteria and needed no further evaluation. Another five articles were independently reviewed by two of the authors, after which those by Hammar et al (21), Sihm et al (22), and Suadicani et al (23) were included, while those by Lynch et al (24) and Murphy (25) were excluded since they did not conform closely enough to the demands or the control dimensions of the job-strain model. Another three, by Billing et al (26), Karasek et al (27), and Messner \& Sihm $(28)^{6}$ were omitted since only main effects were assessed, and not job strain in relation to the dependent variable. In four instances two or more papers by the same group of authors were combined. The full set of included articles is included in the bibliography.

\section{Assessment of methodological quality ${ }^{7}$ \\ Internal validity assessment}

We reviewed all studies fulfilling the aforementioned criteria with respect to their methodological strengths and weaknesses, focusing upon the aspects that would seriously compromise the internal validity of the reported results. The 15 validity criteria grouped into four categories were derived from the work of Stock (29). They are described in the appendix. The first category, assembly of the sample, includes the avoidance of selection bias, the avoidance of nonresponse bias, and the application of appropriate exclusion criteria. The validity of exposure variable assessment was evaluated by five criteria related to the assessment of point exposure to high psychological demands and to low control, the avoidance of recall bias, the analysis of job strain, the adequate range of variation, and the assessment of temporal aspects of exposure. The four criteria under the category for confounding and effect modification were adjustment for relevant demographic confounders, adjustment of relevant biomedical and behavioral confounders, appropriate consideration of gender as an effect modifier, and assessment of other dimensions of the work environment. The validity criteria for the outcome variable were related to the assessment of the endpoint itself, whether the assessment of outcome was blinded with respect to exposure status, and the adequate range of variation of the outcome variable.

In most cases, the maximum score was 3 points (optimal). For six of the criteria, there was a possibility for

Each of these studies ( 2 case-control, 1 cross-sectional) had some positive results, such that their omission does not represent a bias towards positive findings. However, because of a substantial number of methodological weaknesses, including confounding (26), low response rates (28), and problems with the assessment of the temporal aspects of exposure $(26,28)$ and with countermeasures against recall bias $(26-28)$, among others, the positive results do not contribute major supporting evidence for the job strain hypothesis.

$7 \quad$ The internal validity and directionality ratings were performed separately from our review of the results of the studies. 
4 points, insofar as the authors had used innovative methods that served to advance the state of the art in this field of investigation. Thus the maximum total score possible was 51 . The minimum score for each criterion was usually 1 . There was the possibility of a score of 0 for situations that would seriously undermine the valid- ity of a study's conclusions. Two of us independently assessed each of the studies with respect to the 15 validity criteria. In cases of disagreement, a third served as arbiter.

The methodological ratings for each study are presented in tables $2-5$ according to the 15 internal validity

Table 2. Internal validity criteria for the assembly of the sample, rated according to the appendix. $(\mathrm{CAD}=$ coronary artery disease, $\mathrm{CHD}=$ coronary heart disease, $\mathrm{CVD}=$ cardiovascular disease, $\mathrm{ECG}=$ electrocardiography, $\mathrm{IHD}=$ ischemic heart disease, $\mathrm{MI}=$ myocardial infarction, MONICA = monitoring of trends and developments of cardiovascular disease, NHANES = National Health and Nutrition Examination Survey)

\begin{tabular}{|c|c|c|c|}
\hline \multirow[t]{3}{*}{ Study } & \multicolumn{3}{|c|}{ Internal validity criteria for assembly of the sample } \\
\hline & Avoidance of selection bias & Avoidance of nonresponse bias & $\begin{array}{l}\text { Appropriate clinical exclusion criteria } \\
\text { applied }\end{array}$ \\
\hline & Score \& comment & Score \& comment & Score \& comment \\
\hline \multicolumn{4}{|l|}{ Longitudinal studies } \\
\hline $\begin{array}{l}\text { Alfredsson et al, } \\
1985(30)\end{array}$ & $\begin{array}{l}3 \text { All working men and women in } \\
\text { Stockholm }\end{array}$ & 3 & $\begin{array}{l}1 \text { Previous MI occurrence, cerebrovascu- } \\
\text { lar accidents not excluded at baseline }\end{array}$ \\
\hline $\begin{array}{l}\text { Alterman et al, } \\
1994 \text { (31) }\end{array}$ & $\begin{array}{l}267 \% \text { participation, worker-based, } \\
\text { vital status follow-up } 100 \%\end{array}$ & $\begin{array}{l}267 \% \text { participation, nonrespondents } \\
\text { described }\end{array}$ & 3 \\
\hline $\begin{array}{l}\text { Bosma et al, } 1997 \\
\text { (32); Bosma et al, } \\
\text { 1998 (33); Bosma } \\
\text { et al, } 1998 \text { (34) }\end{array}$ & $\begin{array}{l}2 \text { Worker based cohort: } 79 \% \text { participa- } \\
\text { tion at phase } 2 ; 83 \% \text { in phase } 3 \text {; } \\
\text { subjects with low job control had low- } \\
\text { er participation rates in phase } 2 \text { or } 3\end{array}$ & $\begin{array}{l}273 \% \text { response rate, broken down by } \\
\text { employment grade }\end{array}$ & $\begin{array}{l}3 \text { Excluded IHD by clinical exami- } \\
\text { nation with ECG, at baseline }\end{array}$ \\
\hline $\begin{array}{l}\text { Hall et al, } \\
1993(35)\end{array}$ & $\begin{array}{l}3 \text { Random population sample, } \\
\text { complete follow-up }\end{array}$ & $\begin{array}{l}280 \% \text { response rate, but no descrip- } \\
\text { tion of nonrespondents }\end{array}$ & $\begin{array}{l}1 \text { CVD morbidity assessed at baseline by } \\
\text { self-report, but not excluded or taken } \\
\text { into account }\end{array}$ \\
\hline $\begin{array}{l}\text { Hammar et al, } \\
1994(21) ; \\
\text { Hammar et al, } \\
1998(36)\end{array}$ & $\begin{array}{l}3 \text { Population-based, studied incident } \\
\text { cases with nested case-control design, } \\
\text { controls chosen at time of case inciden- } \\
\text { ce-incidence density sampling }\end{array}$ & 3 Data linkage, implied $100 \%$ & $\begin{array}{l}3 \text { Linked hospital records to personal } \\
\text { identification number ensured that the } \\
\text { incident MI was the first hospitalized } \\
\text { Ml for patients and ruled out hospitalized } \\
\text { Ml for controls }\end{array}$ \\
\hline $\begin{array}{l}\text { Hlatky et al, } \\
1995 \text { (37) }\end{array}$ & $\begin{array}{l}0 \text { Evidence for selection attrition (stop- } \\
\text { ped working) among those exposed } \\
\text { to job strain or low control and likely } \\
\text { selection bias in assembly of original } \\
\text { sample }\end{array}$ & $\begin{array}{l}399 \% \text { response rate in Mark et al, } \\
1992(49)\end{array}$ & $\begin{array}{l}3 \text { Severe organic heart disease } \\
\text { excluded and CHD status at } \\
\text { baseline taken into account }\end{array}$ \\
\hline $\begin{array}{l}\text { Johnson et al, } \\
1989(38)\end{array}$ & $\begin{array}{l}3 \text { Population-based cohort, complete } \\
\text { follow-up }\end{array}$ & $\begin{array}{l}280 \% \text { participation, nonrespondents } \\
\text { not described }\end{array}$ & $\begin{array}{l}1 \text { CVD morbidity assessed at baseline } \\
\text { by self-report, but not excluded or } \\
\text { taken into account }\end{array}$ \\
\hline $\begin{array}{l}\text { Johnson et al, } \\
1996(39)\end{array}$ & $\begin{array}{l}3 \text { Population-based cohort with } \\
\text { nested case-control design, } \\
\text { complete follow-up }\end{array}$ & $\begin{array}{l}280 \% \text { participation, nonrespondents } \\
\text { not described }\end{array}$ & $\begin{array}{l}1 \text { CVD morbidity assessed at baseline } \\
\text { by self-report, but not excluded or } \\
\text { taken into account }\end{array}$ \\
\hline $\begin{array}{l}\text { Karasek et al, } \\
1981(40)\end{array}$ & $\begin{array}{l}2 \text { Population-based random sample, } \\
\text { nested case-control design-but not } \\
\text { incidence-density-sampled controls }\end{array}$ & $\begin{array}{l}292 \% \text { initial response rate, non- } \\
\text { respondents not described }\end{array}$ & 2 Adjusted for self-report of CVD \\
\hline $\begin{array}{l}\text { Kivimäki et al, } \\
2002(41)\end{array}$ & $\begin{array}{l}3 \text { Worker-based with } 100 \% \\
\text { follow-up of vital status of cohort }\end{array}$ & $\begin{array}{l}1 \text { Refusals replaced by others on } \\
\text { list, no description or figures given }\end{array}$ & $\begin{array}{l}3 \text { Clinical evaluation performed, CVD at } \\
\text { baseline excluded }\end{array}$ \\
\hline $\begin{array}{l}\text { Kuper \& Marmot, } \\
2003(42)\end{array}$ & $\begin{array}{l}3 \text { Worker-based sample with } 99.9 \% \\
\text { follow-up of vital status, } 75.9 \% \\
\text { follow-up for morbidity in phase } 5\end{array}$ & $\begin{array}{l}273-77 \% \text { nonrespondents broken } \\
\text { down by employment grade }\end{array}$ & $\begin{array}{l}3 \text { IHD excluded by clinical examination, } \\
\text { with ECG }\end{array}$ \\
\hline $\begin{array}{l}\text { Lee et al, } 2002 \\
\text { (43) }\end{array}$ & $\begin{array}{l}2 \text { Disease-free working survivors } \\
16 \text { years after the initiation of a } \\
\text { worker-based (registered nurses) } \\
\text { cohort study, } 95.5 \% \text { follow-up }\end{array}$ & $\begin{array}{l}278 \% \text { responded, several character- } \\
\text { istics of nonresponders described }\end{array}$ & $\begin{array}{l}3 \text { Those who reported } \mathrm{CHD} \\
\text { excluded, diagnosis confirmed by } \\
\text { review of medical records }\end{array}$ \\
\hline $\begin{array}{l}\text { Orth-Gomér et al, } \\
2000(44)\end{array}$ & $\begin{array}{l}2 \text { Hospital-based selection, clear } \\
\text { diagnostic inclusion criteria (acute } \\
\text { MI or unstable angina pectoris), } \\
<65 \text { years of age; return to work } \\
\text { in relation to job strain does not } \\
\text { appear to have been assessed }\end{array}$ & $\begin{array}{l}243 / 335(13 \%) \text { nonrespondents, } 200 \text { par- } \\
\text { ticipants were working at the time of the } \\
\text { examination } 3-6 \text { months after the event; } \\
\text { nonrespondents included } 13 \text { who were } \\
\text { too sick and } 21 \text { who declined for other rea- } \\
\text { sons, including inability to speak Swedish; } \\
\text { no comparison of response rate between } \\
\text { those with and without recurrent events }\end{array}$ & $\begin{array}{l}3 \text { Endpoints were recurrent } \mathrm{MI} \text { and } \\
\text { mortality, caseness based on } \\
\text { hospital and death registers }\end{array}$ \\
\hline $\begin{array}{l}\text { Reed et al, } 1989 \\
\text { (45) }\end{array}$ & $\begin{array}{l}3 \text { Population-based with complete } \\
\text { follow-up of cohort }\end{array}$ & $\begin{array}{l}2 \text { 9878/11148, 89\% initial response } \\
\text { rate, nonrespondents not described }\end{array}$ & $\begin{array}{l}3 \text { CHD excluded, implied by ex- } \\
\text { amination }\end{array}$ \\
\hline $\begin{array}{l}\text { Steenland et al, } \\
1997(46)\end{array}$ & $\begin{array}{l}3 \text { Population based sample, } 93 \% \\
\text { follow-up }\end{array}$ & $\begin{array}{l}2 \text { NHANES response rate } 70 \% \text {, non- } \\
\text { respondents described }\end{array}$ & 2 Self-reported CVD \\
\hline
\end{tabular}


Table 2. Continued

\begin{tabular}{|c|c|c|c|c|}
\hline \multirow[t]{3}{*}{ Study } & \multicolumn{4}{|c|}{ Internal validity criteria for assembly of the sample } \\
\hline & \multicolumn{2}{|c|}{ Avoidance of selection bias } & \multirow[t]{2}{*}{ Avoidance of nonresponse bias } & \multirow[t]{2}{*}{$\begin{array}{l}\text { Appropriate clinical exclusion } \\
\text { criteria applied }\end{array}$} \\
\hline & & ore \& comment & & \\
\hline $\begin{array}{l}\text { Suadicani et al, } \\
1993(23)\end{array}$ & & $\begin{array}{l}\text { Survivors from a } 15 \text {-year worker- } \\
\text { based cohort study }\end{array}$ & $\begin{array}{l}175 \% \text { response rate, nonrespondents } \\
\text { not described }\end{array}$ & $\begin{array}{l}3 \text { Self-report confirmed by } \\
\text { hospital records }\end{array}$ \\
\hline $\begin{array}{l}\text { Theorell et al, } \\
1991 \text { (47) }\end{array}$ & 2 & $\begin{array}{l}\text { Hospital based selection- } \\
\text { follow-up of survivors of definite } \\
\text { MI occurring prior to age } 45 \text { years }\end{array}$ & $\begin{array}{l}2116 / 127(91 \%) \text { examined within } 2 \text { weeks; } \\
\text { excluded } 6 \text { not working and } 31 \text { immigrantes for } \\
\text { reasons of language competence; } N=79 \\
\text { followed-up }\end{array}$ & $\begin{array}{l}3 \text { Mortality study, excluded survi- } \\
\text { vors with reinfarction or other } \\
\text { cardiac complications occurring } \\
\text { during follow-up }\end{array}$ \\
\hline \multicolumn{5}{|l|}{ Case-control studies } \\
\hline $\begin{array}{l}\text { Alfredsson et al, } \\
1982 \text { (50); Alfreds- } \\
\text { son \& Theorell, } \\
1983(51)\end{array}$ & 2 & $\begin{array}{l}\text { Population-based, case-control } \\
\text { study }\end{array}$ & 3 & $\begin{array}{l}3 \mathrm{Ml} \text { excluded in controls during } \\
\text { study period }\end{array}$ \\
\hline $\begin{array}{l}\text { Bobák et al, } \\
1998(52)\end{array}$ & 2 & $\begin{array}{l}\text { Population-based but survivor bias } \\
\text { possible due to case-control design }\end{array}$ & $\begin{array}{l}2 \text { 179/191=94\% eligible cases, } 784 / 813 \\
\text { (96\%) eligible controls, but initial } \\
\text { participation rate of controls } 75 \%\end{array}$ & 3 MONICA protocol \\
\hline $\begin{array}{l}\text { Emdad et al, } \\
1997(53)\end{array}$ & 2 & $\begin{array}{l}\text { CHD patients recruited from the } \\
\text { clinic, controls from working } \\
\text { population-survivor bias possible }\end{array}$ & $\begin{array}{l}213 / 21(62 \%) \text { cases, nonrespondents described, } \\
87 / 130(67 \%) \text { noncase professional drivers were } \\
\text { potential participants (based on matching), non- } \\
\text { participant noncases not described }\end{array}$ & $\begin{array}{l}2 \text { Noncases had 2-channel ECG } \\
\text { during protocol and no known } \\
\text { IHD by self-report }\end{array}$ \\
\hline $\begin{array}{l}\text { Hallqvist et al, } 1998 \\
\text { (54); Theorell et al, } \\
1998 \text { (55); Reuter- } \\
\text { wall et al, } 1999 \text { (56); } \\
\text { Peter et al, } 2002 \text { (57) }\end{array}$ & & $\begin{array}{l}\text { Population-based, but survivor } \\
\text { bias possible due to case-control } \\
\text { design }\end{array}$ & $\begin{array}{l}2 \text { Men } 82 \% \text { cases, } 75 \% \text { controls; women } \\
72 \% \text { cases, } 70 \% \text { controls; some } \\
\text { description of nonparticipation among } \\
\text { men and women }\end{array}$ & $\begin{array}{l}3 \text { All available medical records } \\
\text { scrutinized, health examination } \\
\text { of controls performed }\end{array}$ \\
\hline $\begin{array}{l}\text { Netterstrøm et al, } \\
1999(58)\end{array}$ & 2 & $\begin{array}{l}\text { Consecutive MI cases in two } \\
\text { university hospitals, random } \\
\text { population sample for controls }\end{array}$ & $\begin{array}{l}2100 \% \text { for cases, } 90 \% \text { controls, non- } \\
\text { respondents of the latter not described }\end{array}$ & $\begin{array}{l}1 \text { No explicit mention that MI } \\
\text { ruled out for controls }\end{array}$ \\
\hline $\begin{array}{l}\text { Sihm, Dehlholm } \\
\text { et al,1991 (22) }\end{array}$ & 2 & $\begin{array}{l}\text { Hospitalized MI survivors, hospi- } \\
\text { tal and population based controls }\end{array}$ & $\begin{array}{l}2 \text { 52/54 (96\%) eligible cases, } \\
72 / 86(84 \%) \text { eligible controls, non- } \\
\text { respondents not described }\end{array}$ & $\begin{array}{l}3 \text { Excluded controls with ECG signs } \\
\text { of } \mathrm{MI} \text {, or history of angina pec- } \\
\text { toris or intermittent claudication }\end{array}$ \\
\hline $\begin{array}{l}\text { Theorell et al, } \\
1987(59)\end{array}$ & 2 & $\begin{array}{l}\text { Hospitalized MI survivors, } \\
\text { population-based controls }\end{array}$ & $\begin{array}{l}2116 / 127(91 \%) \text { cases examined, } 31 \text { nonnative } \\
\text { Swedes excluded, } 13 \text { patients excluded because } \\
\text { not working; } 116 / 125(95 \%) \text { controls agreed to } \\
\text { participate, nonrespondents not described }\end{array}$ & $\begin{array}{l}3 \text { History, maximal exercise } \\
\text { stress test }\end{array}$ \\
\hline $\begin{array}{l}\text { Wamala et al, } \\
2000(60)\end{array}$ & & $\begin{array}{l}\text { Cases admitted to cardiac clinic } \\
\text { for acute cardiac event, } \\
\text { population-based controls }\end{array}$ & $\begin{array}{l}3 \text { 292/335 (87\%) cases included (of those not in- } \\
\text { cluded } 5 \text { had died); } 82.5 \% \text { controls, controls } \\
\text { compared with random population sample, no } \\
\text { differences in educational or life-style factors }\end{array}$ & $\begin{array}{l}2 \text { No heart disease symptoms, no } \\
\text { hospitalization for previous } \\
5 \text { years among the controls }\end{array}$ \\
\hline $\begin{array}{l}\text { Yoshimasu \& Fuku- } \\
\text { oka Heart Study } \\
\text { Group, } 2001 \text { (61) }\end{array}$ & 2 & $\begin{array}{l}\text { MI cases surviving to rehabilitation, } \\
\text { admitted to } 22 \text { collaborating hospitals, } \\
\text { population-based controls }\end{array}$ & $\begin{array}{l}1435 / 507(86 \%) \text { cases, } 664 / 1325(50 \%) \text { controls, } \\
\text { psychosocial characteristics of nonparticipant } \\
\text { controls described in detail }\end{array}$ & $\begin{array}{l}2 \text { Referents excluded if prior } \\
\text { history of MI, but unclear how } \\
\text { assessed }\end{array}$ \\
\hline \multicolumn{5}{|l|}{ Cross-sectional studies } \\
\hline Hall et al, 1993 (35) & 2 & Population-based, cross-sectional & $\begin{array}{l}280 \% \text { response rate, nonrespondents } \\
\text { not described }\end{array}$ & 3 Cross-sectional study of CVD \\
\hline $\begin{array}{l}\text { Hlatky et al, } 1995 \\
\text { (37) }\end{array}$ & 0 & $\begin{array}{l}\text { Patients coming to tertiary clinical } \\
\text { center for angiography to work-up } \\
\text { chest pain, no diagnostic entity, } 24 \% \\
\text { had normal coronary arteries, } \\
\text { selection bias likely }\end{array}$ & $399 \%$ response rate from Mark et al (49) & $\begin{array}{l}3 \text { Excluded those requiring } \\
\text { intensive cardiac care at the time } \\
\text { of angiography, pericardial or } \\
\text { myocardial disease, baseline } \\
\text { CHD status taken into account }\end{array}$ \\
\hline $\begin{array}{l}\text { Johnson \& Hall, } \\
1988 \text { (5) }\end{array}$ & 2 & $\begin{array}{l}\text { Population-based cross- } \\
\text { sectional study }\end{array}$ & $\begin{array}{l}279 \% \text { and } 81 \% \text {, effects of nonresponse on varia- } \\
\text { bles concerning illness found to be minimal }\end{array}$ & 3 Cross-sectional study of CVD \\
\hline $\begin{array}{l}\text { Johnson et al, } \\
1989 \text { (38) }\end{array}$ & 2 & $\begin{array}{l}\text { Population-based, cross- } \\
\text { sectional study }\end{array}$ & $\begin{array}{l}280 \% \text { response rate, nonrespondents not } \\
\text { described }\end{array}$ & 3 Cross-sectional study of CVD \\
\hline $\begin{array}{l}\text { Karasek et al, } \\
1988(63)\end{array}$ & & $\begin{array}{l}\text { Representative population } \\
\text { sample }\end{array}$ & $\begin{array}{l}2 \text { NHANES—response rate } 70 \% \text {, non- } \\
\text { respondents described }\end{array}$ & 3 \\
\hline $\begin{array}{l}\text { Netterstrøm et al, } \\
1998 \text { (64) }\end{array}$ & & $\begin{array}{l}\text { Population-based, cross- } \\
\text { sectional study }\end{array}$ & $\begin{array}{l}163 \% \text { response rate, nonrespondents not } \\
\text { described }\end{array}$ & 3 \\
\hline $\begin{array}{l}\text { Sacker et al, } \\
2001(65)\end{array}$ & 2 & $\begin{array}{l}\text { Population based, cross- } \\
\text { sectional study }\end{array}$ & 1 Response rate not reported & 3 Cross-sectional study of CVD \\
\hline $\begin{array}{l}\text { Yoshimasu et al, } \\
200(66)\end{array}$ & 0 & $\begin{array}{l}\text { Patients undergoing angiography } \\
\text { for suspected or known IHD, } \\
62 \% \text { did not have significant } \\
\text { CAD, selection bias likely }\end{array}$ & $\begin{array}{l}2733 / 838(87.5 \%) \text { said to have participated in the } \\
\text { study; however, a large number of exclusions } \\
\text { were performed for various reasons, such that } \\
197 \text { men remained in the analysis-no descrip- } \\
\text { tion of nonrespondents or of characteristics of } \\
\text { the large number of those excluded }\end{array}$ & $\begin{array}{l}3 \text { Caseness defined by extent to } \\
\text { coronary artery stenosis, } \\
\text { excluded valvular heart disease }\end{array}$ \\
\hline
\end{tabular}


Table 3. Internal validity criteria for the assessment of the exposure variables rated according to the appendix. (CAD = coronary artery disease, $\mathrm{CHD}=$ coronary heart disease, HANES $=$ Health and Nutrition Examination Survey, HES $=$ Health Examination Survey, JCQ = job content questionnaire, PSJEM = pshychosocial job exposure matrix, $\mathrm{PSJSQ}=$ psychosocial job strain questionnaire, $\mathrm{QES}=$ quality of employment surveys)

\begin{tabular}{|c|c|c|c|c|c|}
\hline \multirow[t]{3}{*}{ Study } & \multicolumn{5}{|c|}{ Internal validity criteria for assessment of the exposure variable } \\
\hline & \multirow{2}{*}{$\begin{array}{l}\text { Valid and reliable } \\
\text { assessment of point } \\
\text { exposure to psycho- } \\
\text { logical demands and } \\
\text { control } \\
\text { Score \& comment }\end{array}$} & \multirow{2}{*}{$\begin{array}{l}\text { Avoidance of recall bias } \\
\text { for the exposure } \\
\text { variable } \\
\text { Score \& comment }\end{array}$} & \multirow{2}{*}{$\begin{array}{l}\text { Analysis of point-expo- } \\
\text { sure to job strain } \\
\text { Score \& comment }\end{array}$} & \multirow{2}{*}{$\begin{array}{l}\text { Adequate range of } \\
\text { variation of the } \\
\text { exposure variable } \\
\text { Score \& comment }\end{array}$} & \multirow{2}{*}{$\begin{array}{l}\text { Valid and reliable assess- } \\
\text { ment of temporal aspects } \\
\text { of exposure } \\
\text { Score \& comment }\end{array}$} \\
\hline & & & & & \\
\hline \multicolumn{6}{|l|}{ Longitudinal studies } \\
\hline $\begin{array}{l}\text { Alfredsson et al, } \\
1985(30)\end{array}$ & $\begin{array}{l}1 \text { Only } 1 \text { item ("hectic } \\
\text { job") for demands } \\
\text { dimension }\end{array}$ & 3 Imputation study & $\begin{array}{l}2 \text { Job strain treated } \\
\text { as a dichotomous } \\
\text { variable }\end{array}$ & 3 & 21 year follow-up \\
\hline $\begin{array}{l}\text { Alterman et al, } \\
1994 \text { (31) }\end{array}$ & 2 Imputed using QES & 3 Imputed & $\begin{array}{l}3 \text { Tertile term and } \\
\text { analysis of multi- } \\
\text { plicative interaction }\end{array}$ & $\begin{array}{l}2 \text { Mainly blue-collar } \\
\text { workers, use of tertile } \\
\text { term only yielded few } \\
\text { exposed to job strain }\end{array}$ & $\begin{array}{l}125 \text {-year follow-up, but } \\
\text { stable occupation of } \\
\text { cohort }\end{array}$ \\
\hline $\begin{array}{l}\text { Bosma et al, } 1997 \\
(32) ; \text { Bosma et al, } \\
1998 \text { (33); Bosma } \\
\text { et al, } 1998 \text { (34) }\end{array}$ & $\begin{array}{l}4 \text { Self-report with White- } \\
\text { White-hall validation } \\
\text { (4 items for demands) } \\
\text { + independent } \\
\text { observer }\end{array}$ & $\begin{array}{l}3 \text { Independent observer, } \\
\text { self-report in phase I, } \\
\text { outcome in phase II } \\
\text { or III }\end{array}$ & $\begin{array}{l}3 \text { Multiplicative inter- } \\
\text { action term calcu- } \\
\text { lated, not predictive } \\
\text { of outcome, tertiles } \\
\text { of control used to } \\
\text { assess dose- } \\
\text { response }\end{array}$ & $\begin{array}{l}2 \text { All employment } \\
\text { grades of white-collar } \\
\text { workers, few with job } \\
\text { strain ( } 14.7 \% \text { males, } \\
17.2 \% \text { females by } \\
\text { self-report, } 11.9 \text { \& } \\
18.8 \% \text { by external } \\
\text { assessment) }\end{array}$ & $\begin{array}{l}3 \text { Exposure assessed } \\
\text { twice at 3-year inter- } \\
\text { vals, follow-up of em- } \\
\text { ployment during study }\end{array}$ \\
\hline Hall et al, 1993 (35) & $\begin{array}{l}1 \text { Imputation using } \\
\text { PSJEM and } 2 \text { items } \\
\text { for demands }\end{array}$ & 3 Imputed & 2 Dichotomous variable & 3 & $\begin{array}{l}27-11 \text { years of follow- } \\
\text { up, exposure duration } \\
\text { assessed, but not } \\
\text { temporal proximity, } \\
\text { includes women aged } \\
60-74 \text { years at baseline }\end{array}$ \\
\hline $\begin{array}{l}\text { Hammar et al, } 1994 \\
\text { (21); Hammar et al, } \\
1998 \text { (36) }\end{array}$ & $\begin{array}{l}1 \text { Imputation, demands } \\
\text { assessed by two items }\end{array}$ & 3 Imputation & $\begin{array}{l}3 \text { All } 4 \text { quadrants } \\
\text { assessed }\end{array}$ & 3 & $\begin{array}{l}2 \text { Occupation coded 1-9 } \\
\text { years before MI (1970- } \\
\text { 1975, incident cases } \\
\text { 1976-1984), exposure } \\
\text { assessed twice-occu- } \\
\text { pationally stable cohort }\end{array}$ \\
\hline $\begin{array}{l}\text { Hlatky et al, } \\
1995 \text { (37) }\end{array}$ & $\begin{array}{l}3 \text { JCQ with } 5 \text { items for } \\
\text { demands, but only } 6 \\
\text { of the } 9 \text { items for de- } \\
\text { cision latitude }\end{array}$ & 3 & $\begin{array}{l}3 \text { Quotient term and } \\
\text { quadrant term }\end{array}$ & 3 & $\begin{array}{l}2 \text { No repeated exposure, } \\
\text { 4-year average follow- } \\
\text { up, all employed at } \\
\text { baseline }\end{array}$ \\
\hline $\begin{array}{l}\text { Johnson et al, } \\
1989 \text { (38) }\end{array}$ & $\begin{array}{l}2 \text { Validated question- } \\
\text { naire used, } 2 \text { items } \\
\text { for demands }\end{array}$ & 3 & 3 Quintiles & 3 & $\begin{array}{l}19 \text {-year follow-up, no } \\
\text { assessment of } \\
\text { cumulative exposure }\end{array}$ \\
\hline $\begin{array}{l}\text { Johnson et al, } \\
1996 \text { (39) }\end{array}$ & $\begin{array}{l}1 \text { Imputed \& } 2 \text { items } \\
\text { for demands }\end{array}$ & 3 Imputation & $\begin{array}{l}33 \text { cut points \& some } \\
\text { description of multi- } \\
\text { plicative interaction } \\
\text { analysis }\end{array}$ & 3 & $\begin{array}{l}2 \text { Lifetime exposure } \\
\text { assessed prior to 14- } \\
\text { year follow-up }\end{array}$ \\
\hline $\begin{array}{l}\text { Karasek et al, } \\
1981(40)\end{array}$ & $\begin{array}{l}3 \text { Self-report-2 items } \\
\text { for demands, validat- } \\
\text { ed and expert ratings }\end{array}$ & 3 & $\begin{array}{l}2 \text { Dichotomous } \\
\text { variable }\end{array}$ & 3 & $\begin{array}{l}1 \text { No repeated exposure } \\
\text { assessment, 9-year } \\
\text { follow-up }\end{array}$ \\
\hline $\begin{array}{l}\text { Kivimäki et al, } \\
2002(41)\end{array}$ & $\begin{array}{l}24 \text { items for demands, } \\
12 \text { for decision } \\
\text { latitude, Cronbach } \\
\alpha=0.67 \& 0.78, \\
\text { respectively; however, } \\
\text { some items inconsist- } \\
\text { ent with dimension } \\
\text { (eg, mental strain is } \\
\text { an element of job } \\
\text { control) }\end{array}$ & 3 & $\begin{array}{l}33 \text { levels of exposure } \\
\text { to job strain, de- } \\
\text { mands and decision } \\
\text { latitude }\end{array}$ & $\begin{array}{l}3 \text { Both white-collar and } \\
\text { blue-collar factory } \\
\text { employees }\end{array}$ & $\begin{array}{l}1 \text { Stratified analysis of } \\
\text { employees whose } \\
\text { occupational group } \\
\text { remained unchanged } 5 \\
\text { years after assessment } \\
\text { of exposure to work } \\
\text { stressors but follow-up } \\
\text { of vital status }>25 \\
\text { years }\end{array}$ \\
\hline $\begin{array}{l}\text { Kuper \& Marmot, } \\
2003(42)\end{array}$ & $\begin{array}{l}3 \text { Self-report using } \\
\text { Whitehall demand- } \\
\text { control questionnaire }\end{array}$ & 3 & $\begin{array}{l}33 \text { levels of exposure } \\
\text { to job strain, job } \\
\text { demands, and de- } \\
\text { cision latitude, also } \\
\text { multiplicative inte- } \\
\text { action term }\end{array}$ & $\begin{array}{l}2 \text { White-collar workers } \\
\text { of various grades }\end{array}$ & $\begin{array}{l}2 \text { 11.2-year follow-up, } \\
\text { relied upon baseline } \\
\text { exposure data, but high } \\
\text { correlation between } \\
\text { work characteristics in } \\
\text { phases } 1,2,3 \& 5\end{array}$ \\
\hline
\end{tabular}


Table 3. Continued.

\begin{tabular}{|c|c|c|c|c|c|}
\hline \multirow[t]{3}{*}{ Study } & \multicolumn{5}{|c|}{ Internal validity criteria for assembly of the sample } \\
\hline & \multirow{2}{*}{$\begin{array}{l}\text { Valid and reliable } \\
\text { assessment of point } \\
\text { exposure to psycho- } \\
\text { logical demands and } \\
\text { control } \\
\text { Score \& comment }\end{array}$} & \multirow{2}{*}{$\begin{array}{l}\text { Avoidance of recall bias } \\
\text { for the exposure } \\
\text { variable } \\
\text { Score \& comment }\end{array}$} & \multirow{2}{*}{$\begin{array}{l}\text { Analysis of point-expo- } \\
\text { sure to job strain } \\
\text { Score \& comment }\end{array}$} & \multirow{2}{*}{$\begin{array}{l}\text { Adequate range of } \\
\text { variation of the } \\
\text { exposure variable } \\
\text { Score \& comment }\end{array}$} & \multirow{2}{*}{$\begin{array}{l}\text { Valid and reliable assess- } \\
\text { ment of temporal aspects } \\
\text { of exposure } \\
\text { Score \& comment }\end{array}$} \\
\hline & & & & & \\
\hline Lee et al, $2002(43)$ & $3 \mathrm{JCQ}$ & 3 & $\begin{array}{l}34 \text { quadrants for ex- } \\
\text { posure to job strain, } \\
3 \text { levels of exposure } \\
\text { to demands and } \\
\text { control }\end{array}$ & $\begin{array}{l}1 \text { Narrow single occupa- } \\
\text { tion-84\% registered } \\
\text { nurses, as well as } \\
\text { working former nurses, } \\
\text { although in a variety of } \\
\text { settings (out-patient, } \\
\text { operating room, admin- } \\
\text { istration, variance on } \\
\text { relevant job characteris- } \\
\text { tics not demonstrated) }\end{array}$ & $\begin{array}{l}1 \text { Job strain at baseline } \\
\text { used to categorize } \\
\text { exposure status, } 4 \text {-year } \\
\text { follow-up during } \\
\text { which } 49 \% \text { of } \\
\text { those with job strain at } \\
\text { baseline changed } \\
\text { exposure status }\end{array}$ \\
\hline $\begin{array}{l}\text { Orth-Gomér et al, } \\
2000(44)\end{array}$ & $\begin{array}{l}3 \text { Swedish PSJSQ } 5 \\
\text { items on demands }\end{array}$ & 3 & $\begin{array}{l}3 \text { Ratio calculated and } \\
\text { quartiles used }\end{array}$ & $\begin{array}{l}3 \text { No apparent restric- } \\
\text { tion of occupation }\end{array}$ & $\begin{array}{l}2 \text { Occupationally stable, } \\
\text { median follow-up } 4.8 \\
\text { years }\end{array}$ \\
\hline $\begin{array}{l}\text { Reed et al, } \\
1989 \text { (45) }\end{array}$ & 2 Imputation & 3 & $\begin{array}{l}4 \text { Multiplicative inter- } \\
\text { action term + dose- } \\
\text { response }\end{array}$ & 3 & $\begin{array}{l}1 \text { 18-year follow-up, but } \\
\text { number of years on job } \\
\text { assessed at baseline }\end{array}$ \\
\hline $\begin{array}{l}\text { Steenland et al, } \\
1997 \text { (46) }\end{array}$ & 2 Imputed / QES & 3 & 3 Quadrants & 3 & $\begin{array}{l}0 \text { 12-16 years of follow- } \\
\text { up, single assessment } \\
\text { of employment status } \\
\text { and job characteristics }\end{array}$ \\
\hline $\begin{array}{l}\text { Suadicani et al, } \\
1993 \text { (23) }\end{array}$ & $\begin{array}{l}1 \text { Control = } 1 \text { item, no } \\
\text { mention of validation }\end{array}$ & 3 & 3 Interactions assessed & 3 & $\begin{array}{l}23-4 \text { years of follow-up, } \\
\text { no assessment of } \\
\text { repeated exposure }\end{array}$ \\
\hline $\begin{array}{l}\text { Theorell et al, } \\
1991 \text { (47) }\end{array}$ & $\begin{array}{l}22 \text { questions for de- } \\
\text { mands, influence (3 } \\
\text { items), intellectual dis- } \\
\text { cretion or variety (1 } \\
\text { item each) Swedish } \\
\text { PSJSQ }\end{array}$ & 3 & 3 Quotient term & 3 & $\begin{array}{l}1 \text { All working at baseline, } \\
\text { follow-up time } 6-8 \\
\text { years, all who died } \\
\text { returned to same job }\end{array}$ \\
\hline \multicolumn{6}{|l|}{ Case-control studies } \\
\hline $\begin{array}{l}\text { Alfredsson et al, } \\
1982 \text { (50); Alfreds- } \\
\text { son \& Theorell, } \\
1983(51)\end{array}$ & $\begin{array}{l}1 \text { Imputed and } 1 \text { item } \\
\text { for demands }\end{array}$ & 3 Imputation & $\begin{array}{l}3 \text { Dichotomous ex- } \\
\text { posure, but multi- } \\
\text { plicative interaction in } \\
1983 \text { paper }\end{array}$ & 3 & $\begin{array}{l}1 \text { 0ccupation coded 4-6 } \\
\text { years before MI, no } \\
\text { repeated exposure } \\
\text { assessment }\end{array}$ \\
\hline $\begin{array}{l}\text { Bobák et al, } \\
1998 \text { (52) }\end{array}$ & $\begin{array}{l}3 \text { The Whitehall ques- } \\
\text { tionnaire, } 3 \text { items for } \\
\text { demands, selected by } \\
\text { factor analysis }\end{array}$ & $\begin{array}{l}1 \text { Cases interviewed } \\
2 \text { weeks post-Ml }\end{array}$ & $\begin{array}{l}3 \text { All } 4 \text { quadrants } \\
\text { assessed }\end{array}$ & 3 & $\begin{array}{l}2 \text { Currently employed but } \\
\text { no repeated exposure } \\
\text { assessment }\end{array}$ \\
\hline $\begin{array}{l}\text { Emdad et al, } \\
1997(53)\end{array}$ & 3 Swedish PSJSQ & $\begin{array}{l}1 \text { Case status known to } \\
\text { subject prior to } \\
\text { evaluation of work- } \\
\text { place characteristics }\end{array}$ & 3 Quotient term & $\begin{array}{l}1 \text { Single occupation multi- } \\
\text { variate comparisons be- } \\
\text { tween professional dri- } \\
\text { vers with CHD and con- } \\
\text { trols-professional dri- } \\
\text { vers with hypertension }\end{array}$ & $\begin{array}{l}2 \text { Temporal proximity to } \\
\text { employment among } \\
\text { cases not described, } \\
\text { number of years in } \\
\text { occupation assessed }\end{array}$ \\
\hline $\begin{array}{l}\text { Hallqvist et al, } \\
1998 \text { (54); Theorell } \\
\text { et al, 1998 (55); } \\
\text { Reuterwall et al, } \\
1999 \text { (56); Peter et } \\
\text { al, } 2002 \text { (57) }\end{array}$ & $\begin{array}{l}4 \text { Imputation and self- } \\
\text { report via Swedish } \\
\text { PSJSQ, detailed com- } \\
\text { parison between the } 2 \\
\text { performed for men } \\
3 \text { For women }\end{array}$ & 1 For women & $\begin{array}{l}4 \text { Synergy index for } \\
\text { demands and decision } \\
\text { latitude among men }\end{array}$ & 3 & $\begin{array}{l}4 \text { Cumulative exposure, } \\
\text { all working mainly full- } \\
\text { time within last } 5 \text { years }\end{array}$ \\
\hline $\begin{array}{l}\text { Netterstrøm } \\
\text { et al, } 1999 \text { (58) }\end{array}$ & $\begin{array}{l}24 \text { items for demands } \\
\text { includes physical de- } \\
\text { mands \& threat avoid- } \\
\text { and vigilance } \alpha=0.51 \text {; } \\
6 \text { items for decision } \\
\text { latitude } \alpha=0.65 \& 0.81\end{array}$ & $\begin{array}{l}1 \text { Interview of cases in } \\
\text { the coronary care unit, } \\
\text { by nurses or physi- } \\
\text { cians who likely knew } \\
\text { the caseness, no evi- } \\
\text { dence of overreporting, } \\
\text { but denial not ruled out }\end{array}$ & $\begin{array}{l}34 \text { quadrant } \\
\text { assessment }\end{array}$ & 3 & 2 \\
\hline $\begin{array}{l}\text { Sihm, Dehlholm et al, } 3 \\
1991 \text { (22) }\end{array}$ & $\begin{array}{l}3 \text { Orebro-validated ques- } 1 \\
\text { tionnaire, workload = } \\
\text { quantity of work \& level } \\
\text { of strain (difficulty of } \\
\text { work tasks), also con- } \\
\text { tradictory demands; op- } \\
\text { portunity for personal } \\
\text { development \& growth } \\
\text { ( } 3 \text { items); autonomy }\end{array}$ & $\begin{array}{l}1 \text { Questionnaire ad- } \\
\text { ministered during 1st } \\
\text { week of hospitalization }\end{array}$ & $\begin{array}{l}2 \text { A few dichotomous } \\
\text { combinations }\end{array}$ & 3 & $\begin{array}{l}2 \text { Single assessment, } \\
\text { excluded those on } \\
\text { long-term disability or } \\
\text { sick leave or asked } \\
\text { about current job }\end{array}$ \\
\hline
\end{tabular}


Table 3. Continued.

\begin{tabular}{|c|c|c|c|c|c|}
\hline \multirow[t]{3}{*}{ Study } & \multicolumn{5}{|c|}{ Internal validity criteria for assembly of the sample } \\
\hline & \multirow{2}{*}{$\begin{array}{l}\text { Valid and reliable } \\
\text { assessment of point } \\
\text { exposure to psycho- } \\
\text { logical demands and } \\
\text { control } \\
\text { Score \& comment }\end{array}$} & \multirow{2}{*}{$\begin{array}{l}\text { Avoidance of recall bias } \\
\text { for the exposure } \\
\text { variable } \\
\text { Score \& comment }\end{array}$} & $\begin{array}{l}\text { Analysis of point-expo- } \\
\text { sure to job strain }\end{array}$ & \multirow{2}{*}{$\begin{array}{l}\text { Adequate range of } \\
\text { variation of the } \\
\text { exposure variable } \\
\text { Score \& comment }\end{array}$} & \multirow{2}{*}{$\begin{array}{l}\text { Valid and reliable assess- } \\
\text { ment of temporal aspects } \\
\text { of exposure } \\
\text { Score \& comment }\end{array}$} \\
\hline & & & Score \& comment & & \\
\hline $\begin{array}{l}\text { Theorell et al, } \\
1987 \text { (59) }\end{array}$ & $\begin{array}{l}2 \text { Self-report, } 2 \text { items } \\
\text { for demands, } 1 \text { ques- } \\
\text { tion for variety, } 3 \text { ques- } \\
\text { tions on influence over } \\
\text { work, } 1 \text { question on } \\
\text { intellectual discretion }\end{array}$ & $\begin{array}{l}1 \text { Overreport ruled out, } \\
\text { but not denial }\end{array}$ & 3 Quotient terms & 3 & $\begin{array}{l}1 \text { Cases had been } \\
\text { working at least part- } \\
\text { time, but no explicit } \\
\text { mention of controls, no } \\
\text { repeat exposure, not } \\
\text { clear when question- } \\
\text { naire was administered }\end{array}$ \\
\hline $\begin{array}{l}\text { Wamala et al, } \\
2000(60)\end{array}$ & $\begin{array}{l}3 \text { Swedish PSJSQ as } \\
\text { per Theorell et al (7) }\end{array}$ & 1 & 3 Quotient term & 3 & $\begin{array}{l}2 \text { Excluded those not } \\
\text { currently working, no } \\
\text { repeat exposure } \\
\text { assessment }\end{array}$ \\
\hline $\begin{array}{l}\text { Yoshimasu \& } \\
\text { Fukuoka Heart } \\
\text { Study Group, } 2001 \\
(6 !)\end{array}$ & $\begin{array}{l}2 \text { Japanese version of } \\
\text { the JCQ, question- } \\
\text { naire-based interview, } \\
\text { validated, but only } \\
2 \text { items for demands }\end{array}$ & $\begin{array}{l}1 \text { Self-report within } \\
1 \text { month of acute } \\
\text { MI in cases }\end{array}$ & $\begin{array}{l}3 \text { Used quadrant term, } \\
\text { assessed high, middle } \\
\text { and low strain; also } \\
\text { tested tertile term }\end{array}$ & 3 & $\begin{array}{l}2 \text { Excluded those not } \\
\text { having a full-time job } \\
\text { from job strain } \\
\text { analysis, no repeated } \\
\text { exposure assessment }\end{array}$ \\
\hline \multicolumn{6}{|l|}{ Cross-sectional studies } \\
\hline Hall et al, 1993 (35) & $\begin{array}{l}1 \text { Imputed, } 2 \text { items for } \\
\text { demands }\end{array}$ & 3 & 2 Dichotomous variable & 3 & $\begin{array}{l}3 \text { Measured lifetime } \\
\text { exposure }\end{array}$ \\
\hline $\begin{array}{l}\text { Hlatky et al, } \\
1995 \text { (37) }\end{array}$ & $\begin{array}{l}3 \text { JCQ with } 5 \text { items for } \\
\text { demands, but only } 6 \\
\text { of } 9 \text { latitude items }\end{array}$ & $\begin{array}{l}2 \text { No relation between } \\
\text { angina severity and } \\
\text { job strain, baseline cli- } \\
\text { nical status known to } \\
\text { participant, but appar- } \\
\text { ently not extent of CAD }\end{array}$ & $\begin{array}{l}3 \text { Quotient term \& } \\
\text { quadrant term }\end{array}$ & 3 & $\begin{array}{l}2 \text { Currently employed, no } \\
\text { repeat exposure } \\
\text { assessment }\end{array}$ \\
\hline $\begin{array}{l}\text { Johnson \& Hall, } \\
1988 \text { (5) }\end{array}$ & $\begin{array}{l}2 \text { Self-report, } 2 \text { items for } \\
\text { demands, reproduci- } \\
\text { bility } 0.92, \text { scalability } \\
0.79 ; \text { control } 11 \text { items } \\
\text { Cronbach } \alpha=0.70\end{array}$ & 1 & $\begin{array}{l}4 \text { Synergy index } \\
\text { calculated }\end{array}$ & 3 & $\begin{array}{l}2 \text { No repeat exposure, } \\
\text { currently employed }\end{array}$ \\
\hline $\begin{array}{l}\text { Johnson et al, } \\
1989(38)\end{array}$ & $\begin{array}{l}22 \text { items for demands, } \\
\text { validated questionnaire }\end{array}$ & $\begin{array}{l}1 \text { Exposure and out- } \\
\text { come by self-report } \\
\text { from same interview }\end{array}$ & $\begin{array}{l}3 \text { Dose-response: iso- } \\
\text { strain }\end{array}$ & 3 & $\begin{array}{l}2 \text { Employed at baseline, } \\
\text { no cumulative } \\
\text { exposure }\end{array}$ \\
\hline $\begin{array}{l}\text { Karasek et al, } \\
1988(63)\end{array}$ & 2 Imputed QES & 3 Imputational & $\begin{array}{l}3 \text { Dichotomous, top } \\
20 \% \text {, also analyzed } \\
\text { as a continuous } \\
\text { variable }\end{array}$ & 3 & $\begin{array}{l}1 \text { HES job exposure } \\
\text { assessment } 7-17 \text { years } \\
\text { prior \& HANES 6- } \\
\text { years prior to assess- } \\
\text { ment of outcome }\end{array}$ \\
\hline $\begin{array}{l}\text { Netterstrøm et al } \\
1998(64)\end{array}$ & $\begin{array}{l}3 \text { Whitehall methods, } \\
5 \text { demand items, } \\
13 \text { control }\end{array}$ & $\begin{array}{l}2 \text { Assessed association } \\
\text { between job strain } \\
\text { and other pain, as } \\
\text { well as angina pectoris } \\
\text { and job satisfaction- } \\
\text { no association }\end{array}$ & 34 quadrants & 3 & $\begin{array}{l}2 \text { Currently occupation- } \\
\text { ally active, no repeat } \\
\text { measures }\end{array}$ \\
\hline $\begin{array}{l}\text { Sacker et al, } \\
2001(65)\end{array}$ & $\begin{array}{l}3 \text { Mainly Whitehall JCQ } \\
\text { items: } 6 \text { for job control, } \\
3 \text { for job demands }\end{array}$ & $\begin{array}{l}1 \text { Self-report of ex- } \\
\text { I, posure and outcome } \\
\text { from same interview }\end{array}$ & 34 quadrants & 3 & $\begin{array}{l}2 \text { Currently working full- } \\
\text { time, single assess- } \\
\text { ment of exposure }\end{array}$ \\
\hline $\begin{array}{l}\text { Yoshimasu et al, } \\
2000(66)\end{array}$ & $\begin{array}{l}22 \text { items for demands, } \\
\text { (Cronbach } \alpha=0.61 \text { )], } \\
3 \text { items for control } \\
\text { Cronbach } \alpha=0.54 \text {, } \\
\text { test-retest reliability } \\
0.51\end{array}$ & $\begin{array}{l}2 \text { Excluded those with } \\
\text { previous MI or long- } \\
\text { standing angina } \\
\text { pectoris, question- } \\
\text { naires distributed prior } \\
\text { to angiography, } \\
\text { follow-up blinded } \\
\text { interview either before } \\
\text { or after angiography, } \\
\text { specific instructions } \\
\text { to answer questions } \\
\text { as prior to symptoms } \\
\text { or findings of any } \\
\text { abnormal results } \\
\text { regarding CAD }\end{array}$ & $\begin{array}{l}2 \text { Median cut-points- } \\
10 \% \text { job strain }\end{array}$ & $\begin{array}{l}2 \text { Blue- and white- } \\
\text { collar, no restrictions } \\
\text { on occupation, but } \\
\text { small percentage } \\
\text { exposed to job strain }\end{array}$ & $\begin{array}{l}2 \text { Currently working full- } \\
\text { time, no repeated } \\
\text { exposure assessment }\end{array}$ \\
\hline
\end{tabular}


Table 4. Internal validity criteria for confounding and effect modification according to the appendix. $(B M I=$ body mass index, $C A D=$ coronary artery disease, $\mathrm{CHD}=$ coronary heart disease, $\mathrm{HDL}=$ high-density lipoprotein, $\mathrm{HRT}=$ hormone replacement therapy, $\mathrm{LDL}=\mathrm{low}$ density lipoprotein, SCRF = standard cardiac risk factors, SES = socioeconomic status)

\begin{tabular}{|c|c|c|c|c|c|c|}
\hline \multirow[t]{3}{*}{ Study } & \multicolumn{6}{|c|}{ Internal validity criteria of confounding and effect modification } \\
\hline & \multirow{2}{*}{$\begin{array}{l}\text { Adjustment for relevant } \\
\text { demographic confounders } \\
\text { Score \& comment }\end{array}$} & \multirow{2}{*}{\multicolumn{2}{|c|}{$\begin{array}{l}\text { Adjustment for relevant } \\
\text { biomedical and behavioral } \\
\text { confounders } \\
\text { Score \& comment }\end{array}$}} & Stratification by gender & \multicolumn{2}{|c|}{$\begin{array}{l}\text { Assessment of other } \\
\text { dimensions of the work } \\
\text { environment }\end{array}$} \\
\hline & & & & core \& comment & & core \& comment \\
\hline \multicolumn{7}{|l|}{ Longitudinal studies } \\
\hline $\begin{array}{l}\text { Alfredsson et al, } \\
1985(30)\end{array}$ & $\begin{array}{l}3 \text { Adjusted for nationality, } \\
\text { income \& residence type }\end{array}$ & $\begin{array}{l}2 \text { Adjusted for age, smoking } \\
\text { \& some other biomedical } \\
\text { SCRF }\end{array}$ & 2 & $\begin{array}{l}\text { Stratified analysis, but not } \\
\text { adjusted for HRT or oral } \\
\text { contraceptives }\end{array}$ & & $\begin{array}{l}\text { Explored interaction be- } \\
\text { tween hectic work \& sweaty } \\
\text { work and between hectic } \\
\text { work \& heavy lifting, also } \\
\text { assessed irregular and long } \\
\text { workhours, punctuality, gas } \\
\text { and dust exposure, risk of ex- } \\
\text { plosion, draft }\end{array}$ \\
\hline $\begin{array}{l}\text { Alterman et al, } \\
1994(31)\end{array}$ & $\begin{array}{l}4 \text { Job strain - SES } \\
\text { assessed }\end{array}$ & $\begin{array}{l}3 \text { Strain-biomedical SCRF } \\
\text { interaction done-but no } \\
\text { results, also no strain- } \\
\text { behavioral interaction }\end{array}$ & 3 & & 2 & Occupational class \\
\hline $\begin{array}{l}\text { Bosma et al, } 1997(32) ; \\
\text { Bosma et al, } 1998(33) ; \\
\text { Bosma et al, } 1998 \text { (34) }\end{array}$ & $\begin{array}{l}3 \text { Assessed interaction of } \\
\text { SES and dimensions of } \\
\text { job strain, London-no } \\
\text { assessment of immigrant } \\
\text { status or ethnicity }\end{array}$ & $\begin{array}{l}4 \text { Detailed assessment of be- } \\
\text { havioral factors including } \\
\text { interaction effects; adjust- } \\
\text { ment for smoking, choleste- } \\
\text { rol, high blood pressure, } \\
\text { BMI }\end{array}$ & 2 & $\begin{array}{l}\text { Gender-stratified, no } \\
\text { mention of oral contracep- } \\
\text { tive, HRT, menopause }\end{array}$ & 3 & $\begin{array}{l}\text { Effort-reward imbalance, } \\
\text { social support, employment } \\
\text { grade }\end{array}$ \\
\hline Hall et al, 1993 (35) & $\begin{array}{l}4 \text { Interaction between SES } \\
\text { \& job characteristics }\end{array}$ & 2 Age-adjusted & 2 & $\begin{array}{l}\text { Only women, no adjust- } \\
\text { ment for oral contracep-, } \\
\text { tives, HRT, menopause, } \\
\text { LDL, fibrinogen }\end{array}$ & 2 & Occupational class \\
\hline $\begin{array}{l}\text { Hammar et al, } \\
1994 \text { (21); Hammar } \\
\text { et al, } 1998(36)\end{array}$ & 3 Adjustment for SES & 2 Age-adjusted & 2 & $\begin{array}{l}\text { No HRT, oral contracep- } \\
\text { tives, menopause, LDL, } \\
\text { fibrinogen }\end{array}$ & 3 & $\begin{array}{l}\text { Interaction assessment for } \\
\text { social support, long work- } \\
\text { hours, noise all by imputa- } \\
\text { tion, occupational class }\end{array}$ \\
\hline Hlatky et al, 1995 (37) & $\begin{array}{l}1 \text { No adjustment for SES } \\
\text { which differed significantly } \\
\text { by CAD severity, no race } \\
\text { or ethnicity adjustment }\end{array}$ & $\begin{array}{l}2 \text { Assume as for cross- } \\
\text { sectional, age, smoking, } \\
\text { diabetes, hypertension, } \\
\text { hypercholesterolemia }\end{array}$ & 1 & $\begin{array}{l}\text { No gender-stratified anal- } \\
\text { ysis, only adjustment, no } \\
\text { mention of HRT, oral con- } \\
\text { traceptives, menopause }\end{array}$ & 3 & $\begin{array}{l}\text { Workhours, physical } \\
\text { demands, occupational status }\end{array}$ \\
\hline $\begin{array}{l}\text { Johnson et al, } \\
1989(38)\end{array}$ & $\begin{array}{l}4 \text { Assessed interaction } \\
\text { between SES \& iso-strain }\end{array}$ & 2 Age-adjusted & 3 & & 2 & $\begin{array}{l}\text { Social support and occupa- } \\
\text { tional class }\end{array}$ \\
\hline $\begin{array}{l}\text { Johnson et al, } \\
1996(39)\end{array}$ & $\begin{array}{l}3 \text { Adjusted for education, } \\
\text { class \& nationality }\end{array}$ & 2 Age, smoking, exercise & 3 & & 3 & $\begin{array}{l}\text { hazards, physical demands, } \\
\text { social support, occupational } \\
\text { status }\end{array}$ \\
\hline Karasek et al, 1981 (40) & 3 Stratified by education & 2 Age, smoking & 3 & & 1 & \\
\hline $\begin{array}{l}\text { Kivimäki et al, } \\
2002 \text { (41) }\end{array}$ & 3 & $\begin{array}{l}2 \text { Physical activity, smoking, } \\
\text { cholesterol, systolic blood } \\
\text { pressure, BMl }\end{array}$ & 2 & $\begin{array}{l}\text { Adjusted for gender, inter- } \\
\text { action effects with work } \\
\text { stressors assessed as not } \\
\text { significant, no stratified } \\
\text { analysis }\end{array}$ & 3 & $\begin{array}{l}\text { Full evaluation of effort- } \\
\text { reward imbalance and } \\
\text { occupational group }\end{array}$ \\
\hline $\begin{array}{l}\text { Kuper \& Marmot, } \\
2003(42)\end{array}$ & $\begin{array}{l}3 \text { Assessed interaction } \\
\text { between SES and job } \\
\text { strain, not race or } \\
\text { ethnicity in London }\end{array}$ & $\begin{array}{l}2 \text { Age, smoking, serum cho- } \\
\text { lesterol, hypertension, } \\
\text { exercise, BMI, alcohol }\end{array}$ & 2 & $\begin{array}{l}\text { Nonsignificant interaction } \\
\text { between job strain and } \\
\text { gender, adjusted but not } \\
\text { stratified, no adjustment } \\
\text { for HRT, menopause }\end{array}$ & 2 & Interaction with SES \\
\hline Lee et al, 2002 (43) & $\begin{array}{l}2 \text { Education, husband's } \\
\text { education, no mention of } \\
\text { race or ethnicity in United } \\
\text { States population }\end{array}$ & $\begin{array}{l}2 \text { Smoking, BMI, hyper- } \\
\text { tension, diabetes, hyper- } \\
\text { cholesterolemia, dietary fat } \\
\text { intake, physical activity, } \\
\text { family history of MI }\end{array}$ & 3 & $\begin{array}{l}\text { Women only, past use of } \\
\text { oral contraceptives, } \\
\text { current use of HRT, } \\
\text { menopausal status }\end{array}$ & 2 & $\begin{array}{l}\text { Nursing type and social } \\
\text { support }\end{array}$ \\
\hline $\begin{array}{l}\text { Orth-Gomér et al, } \\
2000(44)\end{array}$ & $\begin{array}{l}2 \text { Adjusted for education, } \\
\text { not ethnicity in Stockholm }\end{array}$ & $\begin{array}{l}2 \text { Age, standard biomedical } \\
\text { factors, but not behavioral- } \\
\text { no multiplicative interaction } \\
\text { between work \& marital } \\
\text { stress, no mention of home } \\
\text { workhours or children }\end{array}$ & 3 & $\begin{array}{l}\text { Women only, adjusted for } \\
\text { estrogen status }\end{array}$ & 1 & No other job stressors \\
\hline Reed et al, 1989 (45) & $\begin{array}{l}4 \text { Interaction between job } \\
\text { strain \& education, } \\
\text { Japanese language ability }\end{array}$ & 2 Several SCRF & 3 & & 1 & \\
\hline $\begin{array}{l}\text { Steenland et al, } \\
1997(46)\end{array}$ & $\begin{array}{l}2 \text { SES but not race or } \\
\text { ethnicity-United States } \\
\text { study }\end{array}$ & 2 Several SCRF & 3 & & 2 & Occupational status \\
\hline
\end{tabular}


Table 4. Continued.

\begin{tabular}{|c|c|c|c|c|c|c|}
\hline \multirow[t]{3}{*}{ Study } & \multicolumn{6}{|c|}{ Internal validity riteria of confounding and effect modification } \\
\hline & \multicolumn{2}{|r|}{$\begin{array}{l}\text { Adjustment for relevant } \\
\text { demographic confounders }\end{array}$} & $\begin{array}{l}\text { Adjustment for relevant } \\
\text { biomedical and behavioral } \\
\text { confounders }\end{array}$ & \multicolumn{2}{|r|}{ Stratification by gender } & $\begin{array}{l}\text { Assessment of other } \\
\text { dimensions of the work } \\
\text { environment }\end{array}$ \\
\hline & & Score \& comment & Score \& comment & & core \& comment & Score \& comment \\
\hline $\begin{array}{l}\text { Suadicani et al, } \\
1993(23)\end{array}$ & 3 & & $\begin{array}{l}3 \text { Complete assessment of } \\
\text { SCRF + relaxation as a } \\
\text { behavioral variable }\end{array}$ & 3 & & $\begin{array}{l}2 \text { Social support, occupational } \\
\text { status }\end{array}$ \\
\hline $\begin{array}{l}\text { Theorell et al, } \\
1991 \text { (47) }\end{array}$ & 3 & $\begin{array}{l}\text { Adjusted for education, } \\
\text { immigrants excluded }\end{array}$ & $\begin{array}{l}3 \text { Smoking, cholesterol, fam- } \\
\text { ily history, type A behavior, \& } \\
\text { number of stenosed arteries }\end{array}$ & 3 & & $\begin{array}{l}1 \text { No other job stressors } \\
\text { mentioned }\end{array}$ \\
\hline \multicolumn{7}{|l|}{ Case-control studies } \\
\hline $\begin{array}{l}\text { Alfredsson et al, } 1982 \\
\text { (50); Alfredsson \& } \\
\text { Theorell, } 1983 \text { (51) }\end{array}$ & 3 & & 2 Age & 3 & & $\begin{array}{l}3 \text { Shift work, lifting, piece rate, } \\
\text { noise, vibration, accident risk, } \\
\text { overtime work }\end{array}$ \\
\hline Bobák et al, 1998 (52) & 3 & $\begin{array}{l}\text { Adjustment of SES using } \\
\text { various models, but inter- } \\
\text { action effects not assessed }\end{array}$ & $\begin{array}{l}2 \text { Age, hypertension, other } \\
\text { SCRF }\end{array}$ & 3 & & 1 \\
\hline Emdad et al, 1997 (53) & 2 & $\begin{array}{l}\text { No adjustment for race or } \\
\text { ethnicity in Stockholm- } \\
\text { based study }\end{array}$ & $\begin{array}{l}2 \text { Age-adjusted, detailed } \\
\text { assessment of SCRF and } \\
\text { behavioral risk factors, but } \\
\text { not adjusted }\end{array}$ & 3 & & $\begin{array}{l}3 \text { Correlation analysis between } \\
\text { occupational stress index } \\
\text { and dimensions of job strain }\end{array}$ \\
\hline \multirow[t]{2}{*}{$\begin{array}{l}\text { Hallqvist et al, } 1998 \\
\text { (54); Theorell et al, } \\
1998 \text { (55); Reuterwall } \\
\text { et al, } 1999 \text { (56); Peter } \\
\text { et al, } 2002 \text { (57) }\end{array}$} & 3 & $\begin{array}{l}\text { Interaction between job } \\
\text { strain \& social class } \\
\text { among men, no } \\
\text { adjustment for race or } \\
\text { ethnicity in Stockholm }\end{array}$ & $\begin{array}{l}3 \text { Age, smoking, hyper- } \\
\text { tension, lipids, over- } \\
\text { commintment as a } \\
\text { behavioral factor }\end{array}$ & 3 & $\begin{array}{l}\text { Men gender-stratified } \\
\text { analyses }\end{array}$ & $\begin{array}{l}3 \text { Shiftwork, overtime, } \\
\text { supervising, effort-reward } \\
\text { imbalance among men }\end{array}$ \\
\hline & 1 & Women & & 2 & $\begin{array}{l}\text { Women, lipids assessed } \\
\text { but not included in job- } \\
\text { strain risk estimate }\end{array}$ & $\begin{array}{l}2 \text { Effort-reward imbalance } \\
\text { among women }\end{array}$ \\
\hline $\begin{array}{l}\text { Netterstrøm et al, } \\
1999(58)\end{array}$ & 2 & $\begin{array}{l}\text { Employment sector, not } \\
\text { race or ethnicity, } \\
\text { Copenhagen }\end{array}$ & 2 Age, smoking & 3 & & $\begin{array}{l}3 \text { Workhours, moonlighting, } \\
\text { shiftwork, physical demands, } \\
\text { social support, piece work }\end{array}$ \\
\hline $\begin{array}{l}\text { Sihm, Dehlholm et al, } \\
1991 \text { (22) }\end{array}$ & 3 & $\begin{array}{l}\text { No significant difference } \\
\text { in social class, excluded } \\
\text { those with linguistic } \\
\text { problems, Aarhus }\end{array}$ & $\begin{array}{l}3 \text { Age, assessed interactions } \\
\text { between smoking, } \\
\text { cholesterol \& hypertension } \\
\text { on one hand \& workplace } \\
\text { stressors on the other, } \\
\text { patients versus controls }\end{array}$ & 3 & & $\begin{array}{l}3 \text { Job responsibility, job secur- } \\
\text { ity, job sociability, extra re- } \\
\text { sources for help, } 2 \times 2 \text { com- } \\
\text { binations, but no assessment } \\
\text { of workhours, shiftwork, } \\
\text { physical exposures }\end{array}$ \\
\hline Theorell et al, 1987 (59) & & $\begin{array}{l}\text { Education, immigrants } \\
\text { excluded, Stockholm }\end{array}$ & $\begin{array}{l}3 \text { Age-matched, adjusted for } \\
\text { tobacco consumption \& } \\
\text { LDL/HDL; glucose tolerance, } \\
\text { heredity, type-A behavior \& } \\
\text { weight-to-height ratio } \\
\text { assessed not significant in } \\
\text { multiple regression }\end{array}$ & 3 & & 1 \\
\hline Wamala et al, 2000 (60) & 3 & $\begin{array}{l}\text { Detailed exploration } \\
\text { of social class, no } \\
\text { adjustment for ethnicity, } \\
\text { Stockholm }\end{array}$ & $\begin{array}{l}3 \text { Age-matched, adjusted for } \\
\text { smoking, hypertension, } \\
\text { exercise, obesity, lipid status, } \\
\text { hopelessness, coping }\end{array}$ & 3 & $\begin{array}{l}\text { Assessed HRT, adjusted } \\
\text { for menopausal status }\end{array}$ & 2 Occupational class \\
\hline $\begin{array}{l}\text { Yoshimasu \& Fukuoka } \\
\text { Heart Study Group, } \\
2001 \text { (61) }\end{array}$ & 1 & $\begin{array}{l}\text { Percentage blue-collar } \\
\text { jobs lower in nonstrain, } \\
\mathrm{P}=0.13 \text {, occupational } \\
\text { status not included in } \\
\text { multivariate analysis }\end{array}$ & $\begin{array}{l}4 \text { Adjusted for age, hyper- } \\
\text { tension, diabetes, hyper- } \\
\text { lipidemia, angina pectoris, } \\
\text { obesity, cigarette smoking, } \\
\text { alcohol, parental CHD; } \\
\text { assessed interaction of job } \\
\text { strain and type-A behavior }\end{array}$ & 3 & $\begin{array}{l}\text { Examination of job strain } \\
\text { only among men }\end{array}$ & $\begin{array}{l}3 \text { Shift work, social support, } \\
\text { job type }\end{array}$ \\
\hline \multicolumn{7}{|l|}{ Cross-sectional studies } \\
\hline Hall et al, 1993 (35) & 4 & $\begin{array}{l}\text { Assessed interaction be- } \\
\text { tween SES \& job charac- } \\
\text { teristics }\end{array}$ & 2 Age-adjusted & 2 & $\begin{array}{l}\text { No HRT, oral contracep- } \\
\text { tives, menopause, LDL, } \\
\text { fibrinogen }\end{array}$ & 2 Occupational status \\
\hline Hlatky et al, 1995 (37) & 1 & $\begin{array}{l}\text { No adjustment for SES, } \\
\text { this differed significantly } \\
\text { according to CAD, fewest } \\
\text { white-collar workers } \\
\text { among those with } \\
\text { significant CAD }\end{array}$ & $\begin{array}{l}2 \text { Age, smoking, diabetes, } \\
\text { hypertension, cholesterol }\end{array}$ & 1 & $\begin{array}{l}\text { Adjusted for gender but no } \\
\text { gender stratification, women } \\
\text { \& men significantly differed } \\
\text { on outcome, no mention of } \\
\text { HRT, oral contraceptives, } \\
\text { menopausal status }\end{array}$ & $\begin{array}{l}3 \text { Workhours, physical } \\
\text { n demands, occupational } \\
\text { status }\end{array}$ \\
\hline $\begin{array}{l}\text { Johnson \& Hall, } \\
1988(5)\end{array}$ & 4 & $\begin{array}{l}\text { Stratified analysis by so- } \\
\text { cial class, adjustment for } \\
\text { immigrant status }\end{array}$ & 2 Age, smoking, exercise & 2 & $\begin{array}{l}\text { Stratified analysis, no men- } \\
\text { tion of HRT, oral contra- } \\
\text { ceptives, menopause }\end{array}$ & $\begin{array}{l}3 \text { Physical demands adjust- } \\
\text { ment, social support } \\
\text { interaction assessed }\end{array}$ \\
\hline
\end{tabular}


Table 4. Continued.

\begin{tabular}{|c|c|c|c|c|}
\hline \multirow[t]{3}{*}{ Study } & \multicolumn{4}{|c|}{ Internal validity riteria of confounding and effect modification } \\
\hline & $\begin{array}{l}\text { Adjustment for relevant } \\
\text { demographic confounders }\end{array}$ & $\begin{array}{l}\text { Adjustment for relevant } \\
\text { biomedical and behavioral } \\
\text { confounders }\end{array}$ & Stratification by gender & $\begin{array}{l}\text { Assessment of other } \\
\text { dimensions of the work } \\
\text { environment }\end{array}$ \\
\hline & Score \& comment & Score \& comment & Score \& comment & Score \& comment \\
\hline $\begin{array}{l}\text { Johnson et al, } \\
1989 \text { (38) }\end{array}$ & $\begin{array}{l}4 \text { Interaction between SES } \\
\text { and iso-strain }\end{array}$ & 2 Age-adjusted & 3 & 2 Occupational status \\
\hline $\begin{array}{l}\text { Karasek et al, } 1988 \\
\text { (63) }\end{array}$ & 3 Education and race & $\begin{array}{l}2 \text { Age, smoking, systolic } \\
\text { blood pressure }\end{array}$ & 3 & 2 Physical demands \\
\hline $\begin{array}{l}\text { Netterstrøm et al, } \\
1998 \text { (64) }\end{array}$ & $\begin{array}{l}2 \text { SES adjusted, but not } \\
\text { ethnicity or immigrant } \\
\text { status, Copenhagen }\end{array}$ & $\begin{array}{l}2 \text { Age, smoking, systolic } \\
\text { blood pressure, HDL-to- } \\
\text { total cholesterol ratio }\end{array}$ & $\begin{array}{l}2 \text { Stratified analysis was } \\
\text { done for men, not possi- } \\
\text { ble for women because } \\
\text { of empty cell }\end{array}$ & $\begin{array}{l}3 \text { Workhours, social status, } \\
\text { social support, job security }\end{array}$ \\
\hline Sacker et al, 2001 (65) & $\begin{array}{l}2 \text { SES assessed, but not } \\
\text { ethnicity, race, or immi- } \\
\text { grant status }\end{array}$ & $\begin{array}{l}2 \text { Extensive assessment of } \\
\text { standard cardiac risk } \\
\text { factors but not behavior } \\
\text { unrelated to these }\end{array}$ & 3 & $\begin{array}{l}2 \text { Blue-collar versus white- } \\
\text { collar }\end{array}$ \\
\hline $\begin{array}{l}\text { Yoshimasu et al, } \\
2000(66)\end{array}$ & $\begin{array}{l}3 \text { Adjusted for job type as } \\
\text { an indicator of SES }\end{array}$ & $\begin{array}{l}4 \text { Type-A behavior inter- } \\
\text { action assessed, age, stan- } \\
\text { dard biomedical risk- } \\
\text { factor adjustment }\end{array}$ & 3 & $\begin{array}{l}2 \text { Workhours, blue-collar work, } \\
\text { social support }\end{array}$ \\
\hline
\end{tabular}

Table 5. Internal validity criteria for the outcome variable according to the appendix. (CAD = coronary artery disease, $\mathrm{CHD}=$ coronary heart disease, $\mathrm{CPK}=$ creatine phosphokinase, $\mathrm{CVD}=$ cardiovascular disease, $\mathrm{ECG}=$ electrocardiography, HANES $=$ Health and Nutrition Examination Survey, $\mathrm{HES}=$ Health Examination Survey, IHD $=$ ischemic heart disease, $\mathrm{MI}=$ myocardial infarction, WHO $=$ Work Health Organization)

\begin{tabular}{|c|c|c|c|}
\hline \multirow[t]{3}{*}{ Study } & \multicolumn{3}{|c|}{ Internal validity criteria for outcome variable } \\
\hline & $\begin{array}{l}\text { Valid assessment of the outcome } \\
\text { variable }\end{array}$ & $\begin{array}{l}\text { Assessment of outcome blinded with } \\
\text { respect to exposure }\end{array}$ & $\begin{array}{l}\text { Adequate range of variation of the } \\
\text { outcome variable }\end{array}$ \\
\hline & Score \& comment & Score \& comment & Score \& comment \\
\hline \multicolumn{4}{|l|}{ Longitudinal studies } \\
\hline Alfredsson et al, 1985 (30) & 2 Hospital registry & 3 Linkage & 2 Hospitalized cases of MI \\
\hline Alterman et al, 1994 (31) & $\begin{array}{l}2 \text { Mortality from death certificates } \\
\text { - main result }\end{array}$ & $\begin{array}{l}3 \text { Linkage, also explicit blinding } \\
\text { in Ostfeld }\end{array}$ & $\begin{array}{l}3 \text { Whole cohort followed-up for } \\
\text { vital status, all cases included }\end{array}$ \\
\hline $\begin{array}{l}\text { Bosma et al, } 1997(32) ; \\
\text { Bosma et al, } 1998(33) ; \\
\text { Bosma et al, } 1998(34)\end{array}$ & 1 Self-report of IHD & $\begin{array}{l}2 \text { Self-report of outcome, but indepen- } \\
\text { dent as well as self-report of exposure }\end{array}$ & 2 IHD survivors \\
\hline Hall et al, 1993 (35) & 2 National Death Registry & 3 Imputation & $\begin{array}{l}3 \text { Whole cohort followed-up for } \\
\text { vital status, all cases included }\end{array}$ \\
\hline $\begin{array}{l}\text { Hammar et al, } 1994(21) \text {; } \\
\text { Hammar et al, } 1998(36)\end{array}$ & $\begin{array}{l}3 \text { Registry data with previous } \\
\text { validation study }\end{array}$ & 3 Imputation & 3 Fatal and nonfatal MI \\
\hline Hlatky et al, 1995 (37) & $\begin{array}{l}1 \text { Unclear how follow-up was carried } \\
\text { out, states "all patients were con- } \\
\text { tacted" at follow-up intervals to } \\
\text { "document out-come" [p 328] }\end{array}$ & $\begin{array}{l}2 \text { Unclear whether self-report of out- } \\
\text { come, not explicitly blinded assess- } \\
\text { ment }\end{array}$ & $\begin{array}{l}3 \text { Presumably all participants } \\
\text { followed-up regardless of } \\
\text { outcome }\end{array}$ \\
\hline Johnson et al, 1989 (38) & 2 Registry data & 3 & $\begin{array}{l}3 \text { Whole cohort followed-up for } \\
\text { vital status, all cases included }\end{array}$ \\
\hline Johnson et al, 1996 (39) & 2 Registry data & 3 Linkage & $\begin{array}{l}3 \text { Whole cohort followed-up for } \\
\text { vital status, all cases included }\end{array}$ \\
\hline Karasek et al, 1981 (40) & 3 Validated death certificate & 3 & $\begin{array}{l}3 \text { All CVD deaths included during } \\
\text { follow-up period }\end{array}$ \\
\hline Kivimäki et al, 2002 (41) & 2 Registry data & 3 Use of registry & $\begin{array}{l}3 \text { CVD mortality, obtained cause of } \\
\text { death for all participants who died } \\
\text { during the follow-up period }\end{array}$ \\
\hline Kuper \& Marmot, 2003 (42) & $\begin{array}{l}3 \text { National registry data for mortality, } \\
\text { clinical records and ECG reviewed } \\
\text { by two trained coders }\end{array}$ & 3 Independent review & 3 Fatal and nonfatal incident CHD \\
\hline Lee et al, 2002 (43) & $\begin{array}{l}3 \text { WHO criteria for MI, death certifi- } \\
\text { cates corroborated by autopsy or } \\
\text { hospital records }\end{array}$ & 3 Explicitly blinded & 3 Nonfatal MI and fatal CHD \\
\hline Orth-Gomér et al, 2000 (44) & $\begin{array}{l}3 \text { Validated hospital and death } \\
\text { registers }\end{array}$ & 3 Based on registry data & $\begin{array}{l}2 \text { Complete follow-up of patients } \\
\text { hospitalized for cardiac events }\end{array}$ \\
\hline Reed et al, 1989 (45) & $\begin{array}{l}3 \text { Panel of physicians reviewed the } \\
\text { medical data }\end{array}$ & 3 & 3 Entire cohort followed-up \\
\hline
\end{tabular}


Table 5. Continued.

\begin{tabular}{|c|c|c|c|}
\hline \multirow[t]{3}{*}{ Study } & \multicolumn{3}{|c|}{ Internal validity criteria for outcome variable } \\
\hline & $\begin{array}{l}\text { Valid assessment of the outcome } \\
\text { variable }\end{array}$ & $\begin{array}{l}\text { Assessment of outcome blinded with } \\
\text { respect to exposure }\end{array}$ & $\begin{array}{l}\text { Adequate range of variation of the } \\
\text { outcome variable }\end{array}$ \\
\hline & Score \& comment & Score \& comment & Score \& comment \\
\hline Steenland et al, 1997 (46) & $\begin{array}{l}2 \text { Hospital records and death } \\
\text { certificates }\end{array}$ & 3 & $\begin{array}{l}3 \text { IHD deaths and hospital discharges } \\
\text { for heart disease }\end{array}$ \\
\hline Suadicani et al, 1993 (23) & $\begin{array}{l}3 \text { Review of death and hospital regis- } \\
\text { try with validity frequently assessed }\end{array}$ & 3 Registry data & 3 Complete follow-up of cohort \\
\hline Theorell et al, 1991 (47) & $\begin{array}{l}2 \text { Cardiologist review of reinfarction } \\
\text { mortality }\end{array}$ & 2 & $\begin{array}{l}2 \text { Excluded from analysis those who } \\
\text { survived a reinfarction }\end{array}$ \\
\hline \multicolumn{4}{|l|}{ Case-control studies } \\
\hline $\begin{array}{l}\text { Alfredsson et al, } 1982(50) ; \\
\text { Alfredsson \& Theorell, } \\
1983(51)\end{array}$ & 2 Hospital and death registry & 3 & 3 All MI, fatal and nonfatal \\
\hline Bobák et al, 1998 (52) & 3 MONICA protocol & 2 & 2 Survivors of $\mathrm{MI}$ \\
\hline Emdad et al, 1997 (53) & 2 Hospitalized cases of IHD events & $\begin{array}{l}3 \text { All data analysis performed in a } \\
\text { blinded fashion }\end{array}$ & 2 Survivors of IHD events \\
\hline $\begin{array}{l}\text { Hallqvist et al, } 1998(54) ; \\
\text { Theorell et al, } 1998(55) ; \\
\text { Reuterwall et al, } 1999(56) ; \\
\text { Peter et al, } 2002 \text { (57) }\end{array}$ & 3 Explicit diagnostic criteria & $\begin{array}{l}3 \text { Data linkage in men } \\
2 \text { Women }\end{array}$ & 3 All MI, fatal and nonfatal \\
\hline Netterstrøm et al, 1999 (58) & $\begin{array}{l}2 \text { Severe chest discomfort or ECG } \\
\text { signs of Ml accompanied by increa- } \\
\text { ed CPK to twice the normal level }\end{array}$ & 2 Implied & 2 Hospitalized survivors of MI \\
\hline $\begin{array}{l}\text { Sihm, Dehlholm et al, } \\
1991(22)\end{array}$ & 2 "Established diagnosis of Ml" & 2 & $\begin{array}{l}2 \text { Hospitalized survivors of } \mathrm{MI} \\
<55 \text { years old }\end{array}$ \\
\hline Theorell et al, 1987 (59) & $\begin{array}{l}3 \text { WHO criteria for definite MI, CAD } \\
\text { by coronary angiography }\end{array}$ & 2 & $\begin{array}{l}2 \text { Hospitalized survivors of } \mathrm{Ml} \\
<45 \text { years old }\end{array}$ \\
\hline Wamala et al, $2000(60)$ & $\begin{array}{l}3 \text { Explicit diagnostic criteria, in- } \\
\text { cluding WHO criteria for MI }\end{array}$ & 3 & $\begin{array}{l}2 \text { Hospitalized survivors of cardiac } \\
\text { events }\end{array}$ \\
\hline $\begin{array}{l}\text { Yoshimasu \& Fukuoka Heart } \\
\text { Study Group, } 2001 \text { (61) }\end{array}$ & $\begin{array}{l}2 \text { Collaborating cardiologists were } \\
\text { responsible for the diagnosis of } \\
\text { acute MI }\end{array}$ & 2 Implied but not explicit & 2 Hospitalized survivors of acute MI \\
\hline \multicolumn{4}{|l|}{ Cross-sectional studies } \\
\hline Hall et al, 1993 (35) & 1 Self-reported CVD & 3 & 2 Survivors only assessed \\
\hline Hlatky et al, 1995 (37) & $\begin{array}{l}3 \text { CAD assessed by coronary angio- } \\
\text { graphy, with clear diagnostic } \\
\text { criteria }\end{array}$ & 2 & $\begin{array}{l}2 \text { Excluded patients with unstable } \\
\text { angina or other conditions requiring } \\
\text { intensive care at time of angiography }\end{array}$ \\
\hline Johnson \& Hall, 1988 (5) & 1 & 1 & 2 Survivors only assessed \\
\hline Johnson et al, 1989 (38) & $\begin{array}{l}1 \text { Self-report of IHD, although inde- } \\
\text { pendent diagnostic system, no } \\
\text { objective evidence }\end{array}$ & 1 Self-reported exposure and outcome & 2 Only survivors assessed \\
\hline Karasek et al, 1988 (63) & $\begin{array}{l}2 \text { HES review by four physicians, } \\
\text { specific ECG, history and blood } \\
\text { chemistry for definite MI, reliability } \\
\text { assessment made, HANES review } \\
\text { of medical records, physical exami- } \\
\text { nation, ECG not always available }\end{array}$ & 3 Data linkage & 2 Only survivors assessed \\
\hline Netterstrøm et al, 1998 (64) & $\begin{array}{l}1 \text { Self-report only via Rose } \\
\text { questionnaire }\end{array}$ & $\begin{array}{l}1 \text { Self-report of exposure and of } \\
\text { outcome }\end{array}$ & 2 Only survivors assessed \\
\hline Sacker et al, 2001 (65) & 1 Self-report & 1 Self-report of exposure and outcome & 2 Survivors of heart disease \\
\hline Yoshimasu et al, 2000 (66) & $\begin{array}{l}3 \text { Explicit diagnostic criteria for } \\
\text { stenosis }\end{array}$ & 3 & $\begin{array}{l}1 \text { Excluded those with long-standing } \\
\text { angina pectoris or previous MI }\end{array}$ \\
\hline
\end{tabular}

criteria given in the appendix, the criteria being grouped according to their categories, assembly of the sample (avoidance of selection bias, avoidance of nonresponse bias, appropriate clinical exclusion criteria applied) in table 2, assessment of the exposure variable (valid and reliable assessment of point exposure to psychological demands and to control, avoidance of recall bias for the exposure variable, analysis of point-exposure to job strain, adequate range of variation of the exposure variable, valid and reliable assessment of temporal aspects of exposure) in table 3, confounding and effect modification (adjustment for relevant demographic confounders, adjustment for relevant biomedical and behavioral confounders, stratification by gender, assessment of other dimensions of the work environment) in table 4, and the outcome variable (valid assessment of the outcome variable, assessment of outcome blinded with respect to exposure, adequate range of variation of the outcome variable) in table 5 . 


\section{Directionality}

We next asked the question of how these various methodological issues would affect the results and in which direction they would most likely be acting. We examined each of the validity criteria from this perspective, delineating situations that would increase the likelihood of obtaining null results and those that could lead to an overestimation of association. These directionality issues roughly followed the order of the internal validity criteria, although there was no precise one-to-one correspondence between them, since some issues may affect the results in either direction, depending on the specific circumstances. There were also instances in which the way a methodological issue might affect the results could not be determined (eg, a low response rate without any description of the nonrespondents).

The issues that were considered to increase the likelihood of obtaining null results included (i) selection bias in the assembly of the sample, if the participants exposed to job strain but without CVD preferentially entered the study; (ii) selective attrition, if those exposed to job strain or related work stressors selectively stopped working during the follow-up period; (iii) survivor bias (healthy worker effect); (iv) nonexclusion of outcome at baseline leading to dilution of the results; (v) use of the imputation method (imprecise) to define job strain, leading to nondifferential misclassification; (vi) one to two items for assessing psychological demands if the imputation method was used, leading to nondifferential misclassification; (vii) use of a dichotomous variable to define job strain, leading to nondifferential misclassification; (viii) a low percentage of exposure to job strain, leading to a loss of power to detect an existing effect; (ix) single occupation or a limited range of variation of exposure; (x) assessment of exposure to job strain temporally distant from the outcome (studies with long follow-up periods without repeated assessment of exposure status); (xi) lack of a gender-stratified analysis; (xii) likely confounding by another factor, if the relationships were in the opposite direction of the tested association or if several important confounders were not taken into account.

The issues that were considered to increase the likelihood of an overestimation of association were (i) selection bias in the assembly of the sample, if the participants exposed to job strain and with CVD preferentially entered the study; (ii) selective attrition, if those not exposed to job strain or related work stressors selectively stopped working during the follow-up period; (iii) information bias if the outcome was known to the participant at the time of the self-report of exposure; (iv) likely confounding by another factor, if the relationships were in the direction of association. An alternative hypothesis is likely to be operative, whereby a factor other than job strain is the true effect modifier: (v) infor- mation bias if the outcome and exposure were both selfreported.

Next, a judgment was made about the overall direction in which the methodological issues were likely to affect the results concerning associations with job strain, as follows: (i) unequivocal bias to the null: several clear and strong biases to the null and no biases to overestimate; (ii) likely bias to the null: a few likely biases to the null and no clear bias to overestimate, (iii) minimal biases: nearly all of the potential sources of bias fully taken into account; (iv) bias possible in both directions; (v) likely bias to overestimate: one or more likely biases to overestimate and no clear bias to the null; and (vi) unequivocal bias to overestimate: several clear and strong biases to overestimate and no biases to the null.

\section{Strength and consistency of the empirical findings with respect to job strain and cardiovascular disease, reviewed in light of the methodological issues affecting the results}

The salient details with respect to the results of each of the reviewed longitudinal, case-control, and cross-sectional studies are presented in tables 6, 7, and 8, respectively. Table 9 provides a summary of the relationships between the results and the direction in which the methodological issues were likely to affect each study.

\section{Longitudinal studies}

The longitudinal studies (21-23, 30-47) had higher mean total validity ratings than the case-control and cross-sectional studies did. The mean scores of the studies among men were almost identical for the positive, nonsignificant positive, and null studies. The null studies had a somewhat lower mean total score for the women than those that were positive. Of the two longitudinal studies with the highest total scores (score 40), one yielded a significant positive effect estimate $(21,36)$, while null results were obtained in the other one (45).

Notwithstanding the high overall methodological quality of these investigations, in all but two, biases towards the null dominated. In 11 of the 17 studies, the biases were unequivocal. Biases towards the null were generally due to the use of the imputation method and long follow-up times, with no re-assessment of exposure or even employment status. Persons close to or even above usual retirement age were included in the baseline sample of several of the studies with protracted follow-up $(21,35,36$, 38-40, 44-46); this inclusion would have attenuated the effect estimates even further. The imputation method is particularly problematic for the psychological demand 
Table 6. Results of the reviewed longitudinal studies. ${ }^{a}(\mathrm{BMI}=$ body mass index, $\mathrm{CAD}=$ coronary artery disease, $\mathrm{CHD}=$ coronary heart disease, $\mathrm{CVD}=$ cardiovascular disease, $\mathrm{HR}=$ hazards ratio, $\mathrm{HRT}=$ hormone replacement therapy, $\mathrm{IHD}=$ ischemic heart disease, $\mathrm{MI}=$ myocardial infarction, NS = nonsignificant, $0=$ observer-rated, $\mathrm{OR}=$ odds ratio, $\mathrm{RR}=$ relative risk, $\mathrm{SBP}=$ systolic blood pressure, $\mathrm{SES}=$ socioeconomic status, SMR = standardized mortality ratio, SR = self-rated, UK = United Kingdom, US = United States, $95 \% \mathrm{Cl}=95 \%$ confidence interval)

\begin{tabular}{llll}
\hline Study & Participants & $\begin{array}{l}\text { Foll- Illness } \\
\text { ow- outcome }\end{array}$ & $\begin{array}{l}\text { Significant positive } \\
\text { associations }\end{array}$ \\
& up & & $\begin{array}{l}\text { Reported nonsignificant, null } \\
\text { (years) }\end{array}$
\end{tabular}

Studies with significant positive results for job strain and CVD

Hospitalized Men: punctuality (age) SMR 121 Men: hectic work (age) NS,

1985 (30) Swedish,

20-64 years

of age, popu-

Hospitalized

4 biases to null: nonlation-based men, $\mathrm{N}=142$ ties to learn new things (age) SMR women: hectic work (age) NS exclusion of previous MI at baseline, imputation method, 1 item for psychological demands, 113 (95\% Cl 104-123), hectic \& monotonous work (age) SMR 118 few possibilities to learn new things (age + income) SMR $\approx 125$ single cut-point; total (95\% Cl 105-150); women: hectic $\&$ monotonous work (age) SMR 164 (95\% Cl 112-233), monotony (age) SMR 128 (95\% Cl 104-157), low influence on workmates (age) SMR 133 (95\% Cl 102-170), low influence on holidays (age) SMR 145 (95\% Cl 114-182)

Hammar et al , $\quad \mathrm{N}=24913$ 1994 (21); men, $\mathrm{N}=3535$ Hammar et al, women, 1998 (36) population controls,

Swedish, 30-64 years of age, nested case-control study
9 First $\mathrm{Ml}$ $(\mathrm{N}=8833$ men, $\mathrm{N}=1175$ women)

(all): high strain work RR 1.21 (95\% Cl 1.08-1.35), low decision latitude RR 1.19 (95\% lities to learn new things RR 1.3 demand, long follow-up Cl 1.13-1.25); men (white-collar): (95\% Cl 0.9-1.9), hectic work \& outcome (temporally hectic work and low influence few possibilities to learn new things distant to exposure); total over workhours RR 1.4 (95\% Cl RR 1.2 (95\% Cl 1.0-1.4); men 1.1-1.8); women (all): hectic (white-collar): hectic work RR 1.0 work and few possibilities to learn $(95 \% \mathrm{Cl} 0.8-1.3)$, few possibilities new things RR $1.3(95 \% \mathrm{Cl} 1.1-$ to learn new things RR $1.2(95 \% \mathrm{C}$ 1.6), hectic work \& low influence 1.0-1.4), hectic work \& few possion work planning RR $1.3(95 \% \mathrm{Cl}$ bilities to learn new things RR 1.2 $1.1-1.6)$, high-strain work RR $\quad(95 \% \mathrm{Cl} 1.0-1.6)$; women (blue$1.23(95 \% \mathrm{Cl} 1.01-1.51)$, low collar): hectic work RR $0.7(95 \% \mathrm{Cl}$ decision latitude RR 1.44 (95\% $0.5-1.1)$, few possibilities to learn Cl 1.25-1.65); women (white- new things RR 2.1 (95\% Cl 0.9collar): few possibilities to learn 4.9); women (white-collar): new things RR 2.3 (95\% Cl 1.2- hectic work RR 1.8 (95\% Cl 0.94.6) (age, county, calendar year) 3.7) (age, county, calendar year)
Johnson et al, 1989 (38)

Karasek et al $1981(40)$
Bosma et al, 1997 (32); Bosma et al, 1998 (33); Bosma et al, 1998 (34)
$\mathrm{N}=7219$ men, 9 CVD mortality Swedish, 25-65
years of age, population based

study

$\mathrm{N}=1461$ men, Swedish, 18-

60 years of age, population-based study (nested case-control,

$\mathrm{N}=66$ controls) $(\mathrm{N}=193)$

so-strain (total group) RR 1.92 (b5\% Cl 1.15-3.21), iso-strain (95\% Cl 0.58-2.96) (age)
(age)

9 CVD \& cere- High psychological demands brovascular OR $4.0(95 \% \mathrm{Cl} 1.2-13.9$, high mortality $(\mathrm{N}=22)$ psychological demands \& low personal schedule freedom OR 4.0 (95\% Cl 1.1-14.4) (age, education, smoking, CHD symptoms matched at baseline)

Low intellectual discretion OR 1.5 (95\% Cl 0.4-5.1), low personal schedule freedom OR 1.7 (95\% Cl 0.6-4.7) (same adjustment as for positive findings)

$\mathrm{N}=6895$ men, $\mathrm{N}=3413$ women, UK, 3555 years of age, civil servants
5.3 New selfreport: angina pectoris OR $1.54(95 \% \mathrm{Cl} 1.05$ - pectoris OR $1.40(95 \% \mathrm{Cl} 0.93-$ $(\mathrm{N}=177$ men, 2.26), low control (SR) \& diag- 2.10$)$, job strain (SR) \& diagnos$\mathrm{N}=151$ wo- $\quad$ nosed IHD OR $1.6(95 \% \mathrm{Cl} 1.01-$ ed IHD OR $1.16(95 \% \mathrm{Cl} 0.70-$ men), diagno- 2.55), low control (SR) \& any 1.94), job strain (0) \& all outsis IHD CHD event OR $1.55(95 \% \mathrm{Cl}$ comes OR $1.03(95 \% \mathrm{Cl} 0.66-$ $(\mathrm{N}=124$ men, 1.20-2.01), low control (0) \& 1.61); women: Iow control (SR) $\mathrm{N}=42$ wo- $\quad$ any CHD event OR $1.43(95 \% \mathrm{Cl}$ \& angina pectoris OR $1.20(95 \%$ men), any $1.09-1.88)$, job strain (SR) \& Cl 0.74-1.92), low control (SR) CHD event any CHD event OR $1.45(95 \%$ \& diagnosed IHD OR $0.85(95 \%$ $(\mathrm{N}=401$ men, $\mathrm{Cl} 1.03-2.06)$; women: low $\mathrm{Cl}$ 0.38-1.87), low control (0) \& $\mathrm{N}=253$ wo- control (SR) \& any CHD event angina pectoris OR $1.46(95 \% \mathrm{Cl}$ men) OR $1.74(95 \% \mathrm{Cl} 1.15-2.64), \quad 0.87-2.43)$, low control (0) \& low control (0) \& any CHD event diagnosed IHD OR $1.48(95 \% \mathrm{Cl}$ OR $1.73(95 \% \mathrm{Cl} 1.14-2.62) \quad 0.53-3.85)$, job strain (SR) \& any (age and follow-up time) validity criteria score 40

\author{
(n)
}

2 biases to null: nonexclusion of CVD at baseline, long follow-up outcome (temporally distant to exposure); total validity criteria score 37 3 biases to null: dichotomous variable to assess job strain, long follow-up outcome (temporally distant to exposure), matching controls by CHD symptoms \& education attenuated associations; total validity criteria score 36

1 bias to null: all whitecollar workers (few with job strain), 1 possible bias to overestimate although authors demonstrated that this is unlikely: information bias from self-report of exposure and outcome; total validity criteria score 39 $1.72)$, job strain (0) \& any CHD event OR 1.22 (95\% Cl 0.80-1.86) (age and follow-up time) 
Table 6. Continued.

\begin{tabular}{|c|c|c|c|c|}
\hline Study & Participants & $\begin{array}{l}\text { Foll- } \\
\text { ow- } \\
\text { up } \\
\text { (years) }\end{array}$ & $\begin{array}{l}\text { Illness } \\
\text { outcome } \\
\text { ) }\end{array}$ & $\begin{array}{l}\text { Significant positive } \\
\text { associations }^{\text {b }}\end{array}$ \\
\hline $\begin{array}{l}\text { Kivimäki et al, } \\
2002 \text { (41) }\end{array}$ & $\begin{array}{l}\mathrm{N}=545 \text { men, } \\
\mathrm{N}=267 \text { wo- } \\
\text { men, Finnish, } \\
\text { metal factory } \\
\text { employees }\end{array}$ & $\begin{array}{c}\text { Mean } \\
25.6\end{array}$ & $\begin{array}{l}\text { CVD mortality } \\
(\mathrm{N}=60 \text { men, } \\
\mathrm{N}=13 \text { wo- } \\
\text { men) }\end{array}$ & $\begin{array}{l}\text { Intermediate job strain HR } 1.64 \\
(95 \% \mathrm{CI} 0.85-3.19) \text {, high job } \\
\text { strain HR } 2.22(95 \% \mathrm{Cl} 1.04- \\
4.73) \text { (age, gender, occupa- } \\
\text { tional group, smoking, physical } \\
\text { activity, SBP, cholesterol, } \\
\text { BMI) }\end{array}$ \\
\hline $\begin{array}{l}\text { Kuper \& Marmot, } \\
2003 \text { (42) }\end{array}$ & $\begin{array}{l}\mathrm{N}=6895 \text { men, } \\
\mathrm{N}=3413 \text { wo- } \\
\text { men, UK, 35- } \\
55 \text { years of } \\
\text { age, civil ser- } \\
\text { vants }\end{array}$ & $\begin{array}{c}\text { Mean } \\
11\end{array}$ & $\begin{array}{l}\text { Incident-vali- } \\
\text { dated CHD }\end{array}$ & $\begin{array}{l}\text { High demand and low control \& } \\
\text { all CHD HR } 1.38 \text { ( } 95 \% \mathrm{Cl} 1.10- \\
1.75) \text { (age, gender, occupa- } \\
\text { tional grade, coronary risk } \\
\text { factors) }\end{array}$ \\
\hline $\begin{array}{l}\text { Theorell et al, } \\
1991 \text { (47) }\end{array}$ & $\begin{array}{l}\mathrm{N}=79 \text { men, } \\
\text { Swedish, } \\
<45 \text { years of } \\
\text { age, employ- } \\
\text { ed, first Ml } \\
\text { survivors }\end{array}$ & $6-8$ & $\begin{array}{l}\text { Mortality from } \\
\text { repeat MI } \\
(\mathrm{N}=13)\end{array}$ & $\begin{array}{l}\text { Demands divided by variety } \\
\text { (univariate) } \mathrm{P}=0.03 \text {, demands } \\
\text { divided by intellectual discretion } \\
\mathrm{P}=0.02 \text { (biomedical risk factors, } \\
\text { education did not differ signifi- } \\
\text { cantly between groups) }\end{array}$ \\
\hline
\end{tabular}

Reported nonsignificant, null or significant negative associations $^{b}$
Methodological issues ${ }^{c}$ and total validity scores ${ }^{d}$

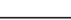

Low job control HR 1.42 (95\% CI 0.72-2.82)(age, gender, occupational group, smoking, physical activity, SBP, cholesterol, BMI)

1 or possibly 2 biases to null: long follow-up temporally distant from exposure, no gender-stratified analysis although no significant interaction with work stressors; no information about nonresponders (unclear how this would affect results); total validity criteria score 37

High demand and low control \& 1 possible bias to null: no fatal CHD or nonfatal MI HR 1.16 gender-stratified analysis $(95 \% \mathrm{Cl} 0.78-1.71)$ (age, although no significant gender, occupational grade, interaction with job strain; coronary risk factors) total validity criteria score 39

Demands NS, single aspects of 1 bias to null: long followdecision latitude NS (univariate) up outcome (temporally distant to exposure diluted associations); 1 possible bias to overestimate: all who died had returned to same work as pre-MI (no mention of survivors, could not rule out that survivors, who as a group had lower job strain exposure selectively, did not return to work); total validity criteria score 35

Studies with positive results for job strain and CVD, but none of which were statistically significant

Alterman et al, $\quad \mathrm{N}=1683$ men, $25 \mathrm{CHD}$ mortality High decision latitude RR 0.76 $1994(31)$

$\mathrm{N}=1683$ me
US, 38-56 High decision latitude RR 0.76 years of age, healthy Chicago Western Electric employees of European ancestry $(74 \%$ bluecollar)

Steenland et al, N=3575 men, 12- Incident heart Job control (highest compared with lowest quartile) OR 0.71 years of age, populationbased study (58\% blue$(\mathrm{N}=519)$ with lowest quartile) OR 0.71 (95\% Cl 0.54-0.93) (age,
education, blood pressure, other coronary risk factors) cholesterol, smoking, alcohol, family history of CVD

Job strain RR $1.40(95 \%$ CI 0.92-2.14), psychological

3 biases to null: imputation method, low percentage 列 1.26), decision latitude RR 0.76 strain, long follow-up (out$(95 \% \mathrm{Cl} 0.59-1.00)$ (education come temporally distant to \& age, SBP, cholesterol, smok- exposure); total validity ing, alcohol, family history of criteria score 38 CVD)

Blue-collar: job strain OR 1.14 (95\% Cl 0.8-1.63), psychological demands OR $0.64(95 \% \mathrm{Cl}$ $0.4-1.03)$, control OR 0.69 $(95 \% \mathrm{Cl} 0.46-1.02)$, high

biases to null, the latter of which seriously threatened the internal validity of the study: imputation, single assessment of job characteristics temporally very (95\% Cl 0.48-0.99); white-collar: distant to exposure would job strain OR $1.05(95 \% \mathrm{Cl} 0.63$ - strongly dilute associa$1.77)$, psychological demands tions; total validity criteria OR $0.93(95 \% \mathrm{Cl} 0.61-1.44)$, score 35 control OR $0.74(95 \% \mathrm{Cl} 0.43-$ 1.26) (as for positive findings) Job strain: second quartile HR 1.53 (95\% Cl 0.58-4.02), upper 2 quartiles HR $1.69(95 \%$ return to work in relation to Cl 0.72-3.98) (age); job strain: job characteristics not ruled second quartile HR 1.33 (95\% out (possible bias), fairly Cl 0.43-4.10), upper 2 quartiles long follow-up outcome HR 1.67(95\% Cl 0.64-4.32) (temporally distant to expo(age, estrogen status, education, sure); no adjustment for diagnosis at index event, symp- marital stress or assesstoms of heart failure, SBP, ment of interaction with job diabetes mellitis, smoking, lipids)

characteristics (uncertain how this affected results) total validity criteria score 
Table 6. Continued.

\begin{tabular}{|c|c|c|c|c|c|c|}
\hline Study & Participants & $\begin{array}{l}\text { Foll- } \\
\text { ow- } \\
\text { up } \\
\text { (years }\end{array}$ & $\begin{array}{l}\text { Illness } \\
\text { outcome } \\
\text { s) }\end{array}$ & $\begin{array}{l}\text { Significant positive } \\
\text { associations }^{\text {b }}\end{array}$ & $\begin{array}{l}\text { Reported nonsignificant, null } \\
\text { or significant negative } \\
\text { associations }^{b}\end{array}$ & $\begin{array}{l}\text { Methodological issues }{ }^{c} \\
\text { and total validity scores }\end{array}$ \\
\hline \multicolumn{7}{|c|}{ Studies with null results for job strain and cardiovascular disease } \\
\hline $\begin{array}{l}\text { Hall et al, } \\
1993 \text { (35) }\end{array}$ & $\begin{array}{l}\mathrm{N}=5921 \text { wo- } \\
\text { men, Swed- } \\
\text { ish, } 45-74 \\
\text { years of age, } \\
\text { random } \\
\text { population } \\
\text { sample }\end{array}$ & $7-11$ & $\begin{array}{l}1 \text { CVD mortality } \\
(\mathrm{N}=182)\end{array}$ & $\begin{array}{l}\text { Work control \& social support } \\
\text { interaction in a multiplicative } \\
\text { manner with occupational-class- } \\
\text { related risk greater than that } \\
\text { attributable to class alone }\end{array}$ & $\begin{array}{l}\text { Blue-collar: psychological de- } \\
\text { mands OR } 0.71 \text { ( } 95 \% \mathrm{Cl} 0.41- \\
1.24) \text {, Iow control OR } 1.07 \\
(95 \% \mathrm{Cl} 0.76-1.51) \text {, job strain } \\
<1 \text {; white-collar: psychological } \\
\text { demand OR } 0.6 \text { ( } 95 \% \mathrm{Cl} 0.28- \\
1.31) \text {, low control OR } 1.4(95 \% \\
\mathrm{Cl} 0.64-3.09) \text {, job strain } \\
<1 \text { (age) }\end{array}$ & $\begin{array}{l}5 \text { biases to null: nonexclu- } \\
\text { sion of CVD morbidity at } \\
\text { baseline, imputation, } 2 \\
\text { items for psychological de- } \\
\text { mands, single cut point for } \\
\text { job strain, follow-up of out- } \\
\text { come temporally distant to } \\
\text { exposure \& inclusion of } \\
\text { those } 60-74 \text { years of age at } \\
\text { baseline; total validity } \\
\text { criteria score } 35\end{array}$ \\
\hline $\begin{array}{l}\text { Hlatky et al } \\
1995 \text { (37) }\end{array}$ & $\begin{array}{l}\mathrm{N}=1132 \text { men, } \\
\mathrm{N}=357 \text { wo- } \\
\text { men, median } \\
\text { age } 52 \text { years, } \\
\text { US, patients } \\
\text { undergoing } \\
\text { coronary } \\
\text { angiography } \\
\text { (88\% white, } \\
60 \% \text { white- } \\
\text { collar) }\end{array}$ & \multicolumn{2}{|c|}{$\begin{array}{c}\text { Mean Incident non- } \\
4 \text { fatal MI }(\mathrm{N}=7 \mathrm{C} \\
\text { cardiac death } \\
(\mathrm{N}=42)\end{array}$} & & 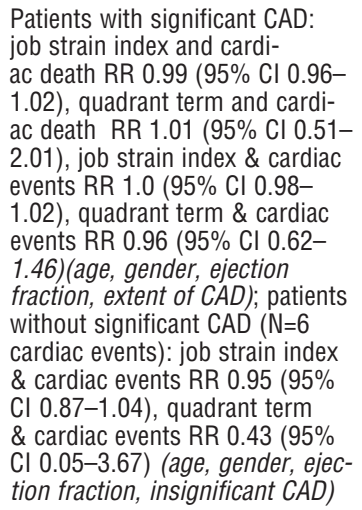 & $\begin{array}{l}4 \text { biases to null: selection } \\
\text { bias likely in assembly of } \\
\text { sample for those exposed } \\
\text { to job strain \& undergoing } \\
\text { angiography but without } \\
\text { CAD, selective attrition of } \\
\text { those exposed to job strain } \\
\text { or low decision latitude, no } \\
\text { gender stratification, con- } \\
\text { founding by SES (job strain } \\
\text { higher for white-collar } \\
\text { workers, but blue-collar } \\
\text { workers had more CAD); } \\
\text { total validity criteria } \\
\text { score } 33\end{array}$ \\
\hline $\begin{array}{l}\text { Johnson et al, } \\
1996 \text { (39) }\end{array}$ & $\begin{array}{l}\mathrm{N}=12517 \text { men, } \\
\text { Swedish, } 25- \\
74 \text { years of } \\
\text { age, popula- } \\
\text { tion-based } \\
\text { nested case-con- } \\
\text { trol study, } \mathrm{N}= \\
2422 \text { controls }\end{array}$ & 14 & $\begin{array}{l}\text { CVD mortality } \\
(\mathrm{N}=521)\end{array}$ & $\begin{array}{l}\text { Low control RR } 1.83 \text { ( } 95 \% \text { Cl } \\
\text { 1.19-2.82), low control \& low } \\
\text { support RR } 2.62 \text { (95\% Cl 1.22-- } \\
5.61) \text { (age, social class, nation- } \\
\text { nality, education, exercise, smok- } \\
\text { ing, last year employed, physi- } \\
\text { cal job demands) }\end{array}$ & $\begin{array}{l}\text { Psychological demands RR } \\
\text { range } 0.88-1.01 \text { ( } 95 \% \mathrm{Cl} 0.66 \\
-1.36) \text {, job strain NS (same } \\
\text { adjustment as for positive } \\
\text { findings) }\end{array}$ & $\begin{array}{l}4 \text { biases to null: nonexclu- } \\
\text { sion of CVD at baseline, } \\
\text { imputation, } 2 \text { items for } \\
\text { psychological demands, } \\
\text { long follow-up outcome } \\
\text { (temporally distant to } \\
\text { exposure); total validity } \\
\text { criteria score } 37\end{array}$ \\
\hline $\begin{array}{l}\text { Lee et al, } \\
2002(43)\end{array}$ & $\begin{array}{l}\mathrm{N}=35038, \mathrm{US} \\
\text { female, regis- } \\
\text { tered nurses, } \\
46-71 \text { years } \\
\text { of age }\end{array}$ & 4 & \multicolumn{2}{|l|}{$\begin{array}{l}\text { Incident, non- } \\
\text { fatal } \mathrm{MI}(\mathrm{N}= \\
108), \text { fatal } \mathrm{CHD} \\
(\mathrm{N}=38)\end{array}$} & $\begin{array}{l}\text { Total CHD: high strain RR } 0.71 \\
(95 \% \text { CI 0.42-1.19) (age, } \\
\text { smoking, alcohol, BMI, hyper- } \\
\text { tension, diabetes, cholesterol, } \\
\text { menopausal status, HRT, aspirin } \\
\text { use, past oral contraceptives, } \\
\text { physical activity, education, } \\
\text { marital status, husband's } \\
\text { education, vitamin E intake, } \\
\text { family history, saturated fat } \\
\text { intake) }\end{array}$ & $\begin{array}{l}3 \text { biases to null: survivor } \\
\text { bias likely in initial sample, } \\
\text { single occupation study lim- } \\
\text { ited range of variation of ex- } \\
\text { posure, assessment of job } \\
\text { strain temporally distant from } \\
\text { outcome; } 49 \% \text { of those ex- } \\
\text { posed to job strain at base- } \\
\text { line changed exposure sta- } \\
\text { tus, but this was not taken } \\
\text { into account in the analyses; } \\
\text { total validity criteria score } 36\end{array}$ \\
\hline $\begin{array}{l}\text { Reed et al, } \\
1989 \text { (45) }\end{array}$ & $\begin{array}{l}\mathrm{N}=4737 \text { men, US } \\
\text { Hawaiians of Ja- } \\
\text { panese descent, } \\
46-65 \text { years } \\
\text { of age, popula- } \\
\text { tion based study }\end{array}$ & & \multicolumn{2}{|c|}{$\begin{array}{l}\text { Incident de- } \\
\text { finite CHD }(\mathrm{N}=359)\end{array}$} & $\begin{array}{l}\text { All calculated forms of job strain } \\
\text { NS, psychological demands NS, } \\
\text { decision latitude NS; in accultur- } \\
\text { ated group: low job strain (vector } \\
\text { score) P<0.05 (age, blood pres- } \\
\text { sure, other coronary risk factors) }\end{array}$ & $\begin{array}{l}2 \text { biases to null: imputation, } \\
\text { very long follow-up out- } \\
\text { come (temporally very } \\
\text { distant to exposure); total } \\
\text { validity criteria score } 40\end{array}$ \\
\hline $\begin{array}{l}\text { Suadicani et al } \\
1993(23)\end{array}$ & $\begin{array}{l}\mathrm{N}=1752 \text { men, } \\
\text { Danish, mean } \\
\text { age } 59.7 \text { years, } \\
\text { survivors from } \\
\text { a } 15 \text {-year } \\
\text { worker-based } \\
\text { cohort study }\end{array}$ & 4 & \multicolumn{2}{|l|}{$\begin{array}{l}\text { Incident first } \\
\text { IHD event } \\
\text { (hospitalized } \\
\text { and fatal } \\
\mathrm{N}=46 \text { ) }\end{array}$} & $\begin{array}{l}\text { Workpace too fast NS, little or } \\
\text { no influence on job organization } \\
\text { NS, monotonous work NS, inter- } \\
\text { actions of the above NS (age, } \\
\text { social class) }\end{array}$ & $\begin{array}{l}2 \text { biases to null: survivor } \\
\text { bias likely in initial sample, } \\
\text { no assessment of occupa- } \\
\text { tional stability \& fairly long } \\
\text { follow-up outcome (tempo- } \\
\text { rally fairly distant to expo- } \\
\text { sure); one item to assess } \\
\text { self-reported job control } \\
\text { (uncertain how this affect- } \\
\text { ed results); total validity } \\
\text { criteria score } 38\end{array}$ \\
\hline
\end{tabular}

\footnotetext{
a All available risk estimates with confidence intervals are shown. For an odds or risk ratio to be considered significant, the $95 \% \mathrm{Cl}$ had to exclude 1.0 .

b The relevant confounders that were either matched between groups or were adjusted are italicized and indicated in parentheses.

${ }^{c}$ Issues that could affect the directionality of the results of each study are given.

d Readers who would like to see additional details about the methodological issues of a particular study or studies may find it helpful to examine the results in this table together with the corresponding validity assessments in tables 2-5.
} 
Table 7. Results of the reviewed case-control referent studies. ${ }^{a}(B M I=$ body mass index, $\mathrm{CHD}=$ coronary heart disease, $\mathrm{CVD}=$ cardiovascular disease, $\mathrm{MI}=$ myocardial infarction, $\mathrm{NS}=$ nonsignificant, $\mathrm{OR}=$ odds ratio, $\mathrm{RR}=$ relative risk, $\mathrm{SES}=$ socioeconomic status, $95 \%$ $\mathrm{Cl}=95 \%$ confidence interval)

\begin{tabular}{|c|c|c|c|}
\hline Study & Participants & $\begin{array}{l}\text { Illness } \\
\text { outcome }\end{array}$ & $\begin{array}{l}\text { Significant positive } \\
\text { associations }^{\mathrm{b}}\end{array}$ \\
\hline \multicolumn{4}{|c|}{ Studies with significant positive results for job strain and CVD } \\
\hline $\begin{array}{l}\text { Alfredsson et al, } \\
1982 \text { (50); } \\
\text { Alfredsson \& } \\
\text { Theorell, } \\
1983 \text { (51) }\end{array}$ & $\begin{array}{l}\text { Swedish men, } \\
<65 \text { years of age, } \\
N=334 \text { cases, } \\
N=882 \text { population } \\
\text { controls }\end{array}$ & $\begin{array}{l}\text { Hospitalized } \\
\text { and or fatal } \\
\mathrm{MI}\end{array}$ & $\begin{array}{l}\text { Total study population: mono- } \\
\text { tony RR } 1.32(95 \% \mathrm{Cl} 1.02- \\
1.70)(\text { age), rushed tempo \& } \\
\text { low influence over work tempo } \\
\text { RR } 1.35(95 \% \mathrm{Cl} 1.01-1.81), \\
(\text { age }) \text {, rushed tempo \& not } \\
\text { learning new things RR } 1.45 \\
(95 \% \mathrm{Cl} 1.02-2.04)(\text { age }) \text {; those } \\
40-54 \text { years of age: hectic work } \\
\& \text { no influence on workpace } \mathrm{RR} \approx \\
1.7(95 \% \mathrm{Cl} \approx 1.3-2.8) \text {, hectic } \\
\text { work \& few possibilities to learn } \\
\text { new things } \mathrm{RR} \approx 2.0 \text { (95\% Cl } \approx 1.3 \\
-3.2) \text { (age \& immigrant status } \\
\text { or education) }\end{array}$ \\
\hline
\end{tabular}

Reported nonsignificant, ull or significant ${ }^{b}$

Rushed tempo RR 1.06 (95\% C 0.82-1.37), low influence over work tempo RR 1.2 (95\% Cl.93 $1.54)$, not learning new things RR 1.19 (95\% Cl 0.93-1.54), rushed tempo \& monotony RR $1.26(95 \% \mathrm{Cl} 0.92-1.72)$ (age) criteria score 38
chalidity cases, $\mathrm{N}=176$ worker controls
Netterstrøm et al, Danish men, $<60 \quad$ Hospitalized Job strain OR $2.3(95 \%$ Cl 1.2 1999 (58) years of age, $\mathrm{N}=76$ acute $\mathrm{MI}$
4.4) (age, employment sector, job category, smoking, social network)
Low decision latitude OR 121 Cl 0.9-2.8) (95\% Cl 0.7-2.1), high psychological demands OR $1.62(95 \%$

3 biases to null: survivo bias as case-control study, MI not explicitly ruled out for controls, outcome known to participants at the time of self-report of exposure (over-report ruled out but not denial), physician \& nurses performed interview-could motivate some patients to deny work stressors if they wanted to return to work; total validity criteria score 32

2 biases to null: survivor

Psychological demands NS, bias as a case-control

Theorell et al, Swedish men, $<45$ Hospitalized Variety of worktasks $\mathrm{P}=0.01$, 1987 (59) years of age, $\mathrm{N}=85$ nonfatal Ml psychological demands divided cases, $\mathrm{N}=116$ community artery athero- psychological demands divided controls matosis (paby intellectual discretion $\mathrm{P}=0.04$ (age, education, alcohol and tobacco consumption,body mass index)

Hallqvist et al, Swedish men, 451998 (54): Peter 64 years of age, et al, 2002 (57); $\mathrm{N}=1047$ cases, $\mathrm{N}=$ Reuterwall et al, 1450 population 1999 (56); $\quad$ controls; Swedish Theorell et al, women, 45-70 1998 (55) years of age, $\mathrm{N}=392$ cases (nonfatal), $\mathrm{N}=533$ population controls tosis and quotient terms or main score 34 $(95 \% \mathrm{Cl} 0.6-3.5)$, psychological other potential sources of
Sihm et al, $1991(22)$ Danish men, $<55 \quad$ Survivors of Heavy workload \& contradictory years of age, $\mathrm{N}=52 \quad \mathrm{Ml}$ cases, $\mathrm{N}=72$

community \& hospital controls
First hospita- Men (all working, self-report): lized or fatal job strain quartile RR 2.2 (95\% $\mathrm{MI}$ or both $\mathrm{Cl} 1.2-4.1)$ [optimal RR 9.2 (95\% Cl 3.3-25.6)], synergy index quartile RR $4.0(95 \% \mathrm{Cl}$ 0.5-30.8) [optimal RR 7.5 (95\% Cl 1.8-30.6)]; men (manual workers, self-report): job strain quartile RR 10.0 (95\% Cl 2.6-38.4) [optimal RR 46.1 (95\% Cl 4.9-429)], synergy index quartile RR 11.1 (95\% C 1.2-107) [optimal RR 23.9 (95\% Cl 2.1-277)], low decision latitude (not imputed) RR $2.3(95 \% \mathrm{Cl} 1.1-4.9)$ (hypertension, smoking, BMI), low decision latitude (imputed) (all working) OR $1.7(95 \% \mathrm{Cl} 1.3$ 2.2) (age, catchment area); women (all, self-report): job strain OR for nonfatal 1.51 (95\% Cl 1.13-2.02) (age, catchment area, demands RR 1.96 (95\% Cl (95 workload \& $0.82(95 \% \mathrm{Cl} 0.54-1.24)$, low ( Cl 1.05-3.02), low workload \& 1.53), contradictory demands good social interaction RR 0.58 RR 1.33 (95\% Cl 0.87-2.02), (95\% Cl 0.35-0.95) (age and low growth \& development RR SES did not differ significantly between patients and controls) tual discretion NS, psychological study, outcome known to demands divided by influence participants at time of selfover workload NS (as for signif- report of exposure (overicant positive); for the patients report ruled out but not the degree of coronary atheroma- denial); total validity criteria effects NS

Men (nonmanual workers, self- 1 bias to null: survivor bias report): job strain quartile RR 1.5 as a case-control study; demands quartile RR $1.2(95 \%$ bias taken into account for $\mathrm{CI} 0.8-1.6)$, low decision latitude men; 1 bias to overestimate RR 1.0 (95\% Cl 0.6-1.7); men (for women): self-report of (manual workers, self report): $\quad$ job characteristics only psychological demands RR 1.2 (outcome known to (95\% CI 0.5-3.1) (hypertension, participants at time of selflatitude (imputed) RR 1.2 (95\% validity criteria score 46 for

Cl 0.8-2.0), negative change in men \& 36 for women decision latitude RR $1.4(95 \%$ C 1.0-2.0) (age, catchment area,

social class, coronary risk

factors)
1 bias to null: survivor bias as a case-control study; 1 bias to overestimate: outcome known to participants at time of self-report of exposure; total validity criteria score 36 0.81 (95\% Cl 0.53-1.24) (as for significant positive) 
Table 7. Continued.

\begin{tabular}{|c|c|c|c|c|c|}
\hline Study & Participants & $\begin{array}{l}\text { Illness } \\
\text { outcome }\end{array}$ & $\begin{array}{l}\text { Significant positive } \\
\text { associations }^{b}\end{array}$ & $\begin{array}{l}\text { Reported nonsignificant, } \\
\text { null or significant }{ }^{b}\end{array}$ & $\begin{array}{l}\text { Methodological issues }{ }^{c} \text { and } \\
\text { total validity scores }{ }^{d}\end{array}$ \\
\hline $\begin{array}{l}\text { Yoshimasu \& } \\
\text { Fukuoka Heart } \\
\text { Study Group, } \\
2001(61)\end{array}$ & $\begin{array}{l}\text { Japanese men, } 40- \\
79 \text { years of age, } \\
\mathrm{N}=173 \text { cases, } \mathrm{N}= \\
303 \text { community } \\
\text { controls }\end{array}$ & $\begin{array}{l}\text { Hospitalized } \\
\text { survivors of } \\
\text { first acute } \\
\text { MI }\end{array}$ & $\begin{array}{l}\text { High job strain OR } 2.2(95 \% \mathrm{Cl} \\
\text { 1.1-4.5) (age, hypertension, } \\
\text { diabetes, hyperlipidemia, angina } \\
\text { pectoris, overweight, cigarette } \\
\text { smoking, alcohol intake, } \\
\text { parental, CHD and shift work) }\end{array}$ & $\begin{array}{l}\text { High job demand RR } 1.3 \text { (95\% } \\
\mathrm{CI} 0.7-2.2) \text {, low job control RR } \\
1.0(95 \% \mathrm{Cl} 0.5-1.7) \text { (as for } \\
\text { significant positive) }\end{array}$ & $\begin{array}{l}1 \text { or possibly } 2 \text { biases to } \\
\text { null: survivor bias case- } \\
\text { control study, those not } \\
\text { exposed to job strain } \\
\text { more frequently blue-collar } \\
\text { ( } \mathrm{P}=.13 \text { ) but occupational } \\
\text { status not included in } \\
\text { multivariate risk estimate; } \\
1 \text { or possibly } 2 \text { biases to } \\
\text { overestimate: nonpartici- } \\
\text { pant referents had signifi- } \\
\text { cantly higher job demands } \\
\text { than participating referents, } \\
\text { outcome known to partici- } \\
\text { pants at time of self-report } \\
\text { of exposure; significantly } \\
\text { more nonparticipant re- } \\
\text { ferents in blue-collar jobs } \\
\text { (unclear how this would } \\
\text { affect results); total validity } \\
\text { criteria score } 33\end{array}$ \\
\hline
\end{tabular}

Studies with positive results for job strain and CVD, but none of which were statistically significant

\begin{tabular}{|c|c|c|c|c|}
\hline $\begin{array}{l}\text { Bobák et al, } \\
1998 \text { (52) }\end{array}$ & $\begin{array}{l}\text { Czech men, } 25-64 \\
\text { years of age, } N= \\
179 \text { cases, } N=784 \\
\text { controls, all full- } \\
\text { time employed }\end{array}$ & $\begin{array}{l}\text { First nonfatal } \\
\text { MI }\end{array}$ & $\begin{array}{l}\text { Highest decision latitude quar- } \\
\text { tile RR } 0.43(95 \% \mathrm{Cl} 0.24-0.79) \\
\text { (age, district, education, hyper- } \\
\text { tension, other coronary risk } \\
\text { factors) }\end{array}$ & $\begin{array}{l}\text { Job strain RR } 1.31 \text { (95\% Cl } \\
0.77-2.25) \text {, highest psycho- } \\
\text { logical demands quartile RR } \\
0.52(95 \% \mathrm{Cl} 0.29-0.93) \text { (as } \\
\text { for the significant positive } \\
\text { findings) }\end{array}$ \\
\hline
\end{tabular}

1 bias to null: survivor bias as a case-control study; 1 possible, though unlikely, bias to overestimate: outcome known to participant at time of self-report of exposure, although the inverse relation to demands argues the opposite-denial; total validity criteria score 35

$\begin{array}{lll}\text { Wamala et al, } & \text { Swedish women, } & \text { Hospitalized Job control } P=0.03 \text {, job strain } \\ 2000(60) & \leq 65 \text { years of age, } & \text { acute Ml or ratio } P=0.02 \text { (age) } \\ N=292 \text { cases, } & \text { unstable an- } \\ & N=292 \text { population } & \text { gina pectoris, } \\ \text { controls } & \text { survivors }\end{array}$

Job control, job strain did not substantially explain the increased CHD risk in the lowest occupational strata

1 attenuated bias to null : survivor bias partially taken into account in assessment of results for those not currently working; 1 bias to over-estimate: outcome known to participants at time of self-report of exposure; total validity criteria score 38

Study with null results for job strain and CVD

Emdad et al, Swedish men, $<52$ Hospitalized

1997 (53) years of age, $\mathrm{N}=13$ ischemic cases, $\mathrm{N}=12$ hyper- heart disease tensive controls, all professional demand NS, decision latitude NS, skill discretion NS, control NS (age)

2 biases to null: survivor bias as a case-control study, single occupation, limited range of variation of exposure; 1 bias to overexposure; 1 bias to overto participant at time of self-report of exposure; total validity criteria score 33

\footnotetext{
a All available risk estimates with confidence intervals are shown. For an odds or risk ratio to be considered significant, the $95 \% \mathrm{CI}$ had to exclude 1.0 .

b The relevant confounders that were either matched between groups or were adjusted are italicized and indicated in parentheses.

${ }^{c}$ Issues that could affect the directionality of the results of each study are given.

d Readers who would like to see additional details about the methodological issues of a particular study or studies, may find it helpful to examine the results in this table together with the corresponding validity assessments in tables 2-5.
} 
Table 8. Results of the reviewed cross-sectional studies. ${ }^{a}(B M I=$ body mass index, $C A D=$ coronary artery disease, $C H D=$ coronary heart disease, $\mathrm{CVD}=$ cardiovascular disease, HANES = Health and Nutrition Examination Survey, HES $=$ Health Examination Survey, $\mathrm{HDL}=$ high-density lipoprotein, $\mathrm{MI}=$ myocardial infarction, $\mathrm{OR}=$ odds ratio, $\mathrm{PR}=$ prevalence ratio, $\mathrm{RR}=$ risk ratio, $\mathrm{SES}=$ socioeconomic status, SOR $=$ standardized odds ratio, $95 \% \mathrm{Cl}=95 \%$ confidence interval)

\begin{tabular}{|c|c|c|c|c|c|}
\hline Study & Participants & $\begin{array}{l}\text { Illness } \\
\text { outcome }\end{array}$ & $\begin{array}{l}\text { Significant positive } \\
\text { associations }^{b}\end{array}$ & $\begin{array}{l}\text { Reported nonsignificant, } \\
\text { null or significant }{ }^{b}\end{array}$ & $\begin{array}{l}\text { Methodological issues and } \\
\text { total validity scores } c, d\end{array}$ \\
\hline \multicolumn{6}{|c|}{ Studies with significant positive results for job strain and CVD } \\
\hline $\begin{array}{l}\text { Karasek et al, } \\
1988 \text { (63) }\end{array}$ & $\begin{array}{l}\text { US men, age } 18- \\
79 \text { years, } N=2409 \\
\text { HES, N=2424 } \\
\text { HANES, population } \\
\text { samples ( } 87 \% \text { \& } \\
88 \% \text { white, } \\
\text { respectively) }\end{array}$ & $\begin{array}{l}\text { MI preva- } \\
\text { lence }(N=39 \\
\text { HES, } N=30 \\
\text { HANES) }\end{array}$ & 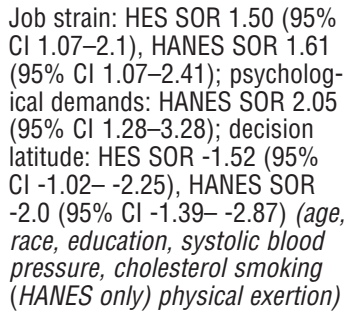 & $\begin{array}{l}\text { Psychological demands HES } \\
\text { SOR } 1.32 \text { ( } 95 \% \mathrm{Cl} 0.91-1.9) \text { (as } \\
\text { for significant positive findings) }\end{array}$ & $\begin{array}{l}3 \text { biases to null: survivor } \\
\text { bias as a cross-sectional } \\
\text { study, imputation, assess- } \\
\text { ment of exposure to job } \\
\text { strain temporally distant } \\
\text { from outcome (7-17 years } \\
\text { prior for HES, } 6 \text { years for } \\
\text { HANES); total validity } \\
\text { criteria score } 36\end{array}$ \\
\hline $\begin{array}{l}\text { Johnson \& Hall, } \\
1988 \text { (5) }\end{array}$ & $\begin{array}{l}\mathrm{N}=7165 \text { men, } \mathrm{N}= \\
6614 \text { women, } \\
\text { Swedish, age } 16- \\
65 \text { years, popula- } \\
\text { tion sample }\end{array}$ & $\begin{array}{l}\text { Self-reported } \\
\text { CVD }(N=409 \\
\text { men, } N=395 \\
\text { women) }\end{array}$ & $\begin{array}{l}\text { Men (blue-collar): high psycho- } \\
\text { logical demands \& low control } \\
\text { PR } 3.55 \text { ( } 95 \% \text { Cl } 1.64-7.69) \text {, } \\
\text { high psychological demands \& } \\
\text { low control \& low support PR } \\
7.22 \text { (95\% Cl 1.6-37.4); men } \\
\text { (white-collar): high psycholog- } \\
\text { ical demands \& low support PR } \\
1.81 \text { (95\% Cl 1.02-3.22), 3- } \\
\text { factor multiplicative interaction } \\
\text { ratio 1.09; women (blue-collar) } \\
\text { high psychological demands \& } \\
\text { low support PR 1.68 (95\% Cl } \\
1.07-2.63) ; \text { women (white- } \\
\text { collar): high psychological } \\
\text { demands \& low support PR } \\
2.06 \text { (95\% Cl 1.05-4.01) (age, } \\
\text { dimensions of "iso-strain") }\end{array}$ & 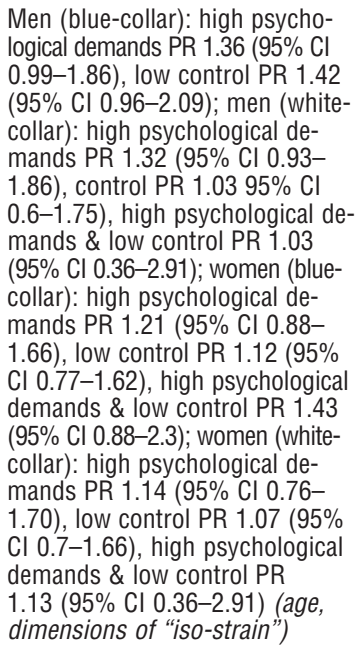 & $\begin{array}{l}1 \text { bias to null: survivor bias } \\
\text { as a cross-sectional study; } \\
2 \text { biases to overestimate: } \\
\text { outcome known to } \\
\text { participants at time of self- } \\
\text { report of exposure, self- } \\
\text { report of exposure \& } \\
\text { outcome; total validity } \\
\text { criteria score } 34\end{array}$ \\
\hline $\begin{array}{l}\text { Johnson et al, } \\
1989 \text { (38) }\end{array}$ & $\begin{array}{l}\mathrm{N}=7219 \text { men, } \\
\text { Swedish, age } 25- \\
65 \text { years, population } \\
\text { sample }\end{array}$ & $\begin{array}{l}\text { Self-reported } \\
\text { CVD }(N=407)\end{array}$ & $\begin{array}{l}\text { All: iso-strain PR } 1.77(95 \% \mathrm{Cl} \\
1.28-2.44) \text {; blue-collar: iso- } \\
\text { strain PR } 2.04 \text { ( } 95 \% \text { Cl 1.24- } \\
\text { 3.36) (age) }\end{array}$ & 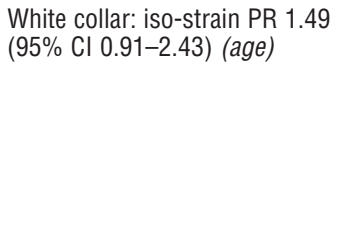 & $\begin{array}{l}1 \text { bias to null: survivor bias } \\
\text { as a cross-sectional study; } \\
2 \text { biases to overestimate: } \\
\text { outcome known to partici- } \\
\text { pants at time of self-report } \\
\text { of exposure, self-report of } \\
\text { exposure \& outcome; to- } \\
\text { tal validity criteria score } 33\end{array}$ \\
\hline $\begin{array}{l}\text { Sacker et al, } \\
2001(65)\end{array}$ & $\begin{array}{l}\mathrm{N}=4235 \text { men, } \\
\text { population-based, } \\
\text { England, age } 20- \\
64 \text { years }\end{array}$ & $\begin{array}{l}\text { Self-reported } \\
\text { heart disease: } \\
\text { angina } 1.1 \% \text {, } \\
\text { possible Ml } \\
6 \% \text {, physi- } \\
\text { cian-diagnos- } \\
\text { ed heart } \\
\text { disease } 5 \% \text {, } \\
\text { any heart } \\
\text { disease } 9 \%\end{array}$ & $\begin{array}{l}\text { High strain: angina OR } 2.46 \\
\text { (95\% Cl 1.23-4.92), possible } \\
\text { MI OR } 1.46 \text { (95\% Cl 1.01-2.12), } \\
\text { physician diagnosed heart } \\
\text { disease OR } 1.50 \text { (95\% Cl 1.02- } \\
2.20) \text {, any heart disease OR } 1.60 \\
\text { (95\% Cl } 1.20-2.13) \text { (age, age }{ }^{2}, \\
\text { SES, diet, smoking, leisure-, } \\
\text { time cholesterol, BMI, diabetes } \\
\text { mellitus, blood pressure }\end{array}$ & & $\begin{array}{l}2 \text { biases to null: survivor } \\
\text { bias as a cross-sectional } \\
\text { study, low percentage } \\
(15 \%) \text { job strain; } 2 \text { biases } \\
\text { to overestimate: outcome } \\
\text { known to participants at } \\
\text { time of self-report of } \\
\text { exposure, self-report of } \\
\text { exposure and outcome; } \\
\text { total validity score } 31\end{array}$ \\
\hline \multicolumn{6}{|c|}{ Studies with positive results for job strain and CVD, but none of which were statistically significante } \\
\hline $\begin{array}{l}\text { Netterstrøm et } \\
\text { al, } 1998(64)\end{array}$ & $\begin{array}{l}\mathrm{N}=512 \text { men, } \mathrm{N}=537 \\
\text { women, Danish, } \\
30-59 \text { years of age, } \\
\text { population-based }\end{array}$ & $\begin{array}{l}\text { Self-reported } \\
\text { angina pecto- } \\
\text { ris }(\mathrm{N}=25 \\
\text { men, } \mathrm{N}=10 \\
\text { women) }\end{array}$ & $\begin{array}{l}\text { Job strain OR } 2.3(95 \% \mathrm{Cl} \\
1.2-4.4) \text { (age, gender, work } \\
\text { hours, psychosocial factors, } \\
\text { social status, smoking, systolic } \\
\text { blood pressure, HDL:cholesterol } \\
\text { ratio) }\end{array}$ & $\begin{array}{l}\text { Men: job strain OR } 2.4(95 \% \mathrm{Cl} \\
0.5-11.5) \text { (age, social status) }\end{array}$ & $\begin{array}{l}1 \text { bias to null: survivor bias } \\
\text { as a cross-sectional study; } \\
2 \text { attenuated biases to over- } \\
\text { estimate: outcome known } \\
\text { to participants at time of } \\
\text { self-report of exposure, } \\
\text { self-report of exposure \& } \\
\text { outcome (however, no } \\
\text { association found between } \\
\text { job strain and other somatic } \\
\text { pains or between job sa- } \\
\text { tisfaction and angina pecto- } \\
\text { ris); low response rate (un- } \\
\text { clear how this affects re- } \\
\text { sults); total validity criteria } \\
\text { score } 32\end{array}$ \\
\hline
\end{tabular}


Table 8. Continued.

\begin{tabular}{|c|c|c|c|c|c|}
\hline Study & Participants & $\begin{array}{l}\text { Illness } \\
\text { outcome }\end{array}$ & $\begin{array}{l}\text { Significant positive } \\
\text { associations }^{b}\end{array}$ & $\begin{array}{l}\text { Reported nonsignificant, } \\
\text { null or significant }{ }^{b}\end{array}$ & $\begin{array}{l}\text { Methodological issues and } \\
\text { total validity scores } c, d\end{array}$ \\
\hline $\begin{array}{l}\text { Yoshimasu et al, } \\
2000(66)\end{array}$ & $\begin{array}{l}\mathrm{N}=197 \text { men, Japan, } \\
\text { undergoing coro- } \\
\text { nary angiography, } \\
\text { but without } \\
\text { long-standing } \\
\text { angina pectoris } \\
\text { or previous MI, } \\
\text { mean age } 54.7 \\
\text { (SD 8.9) years }\end{array}$ & $\begin{array}{l}\text { Presence of } \\
\text { CAD }(\geq 75 \% \\
\text { stenosis of } \geq 1 \\
\text { major coro- } \\
\text { nary arteries } \\
\text { or } \geq 50 \% \text { ste- } \\
\text { nosis of left } \\
\text { main coronary } \\
\text { artery) }\end{array}$ & & $\begin{array}{l}\text { Job strain OR } 1.7 \text { (95\% Cl 0.6- } \\
5.3) \text {, psychological demands } \\
\text { OR } 1.3(95 \% \mathrm{Cl} 0.6-2.6) \text {, low } \\
\text { control OR } 0.8(95 \% \mathrm{Cl} 0.4-1.5) \\
\text { (age, hospital, diabetes, hyper- } \\
\text { lipidemia, overweight, cigarette } \\
\text { smoking, alcohol intake, } \\
\text { parental CHD, job type, hyper- } \\
\text { tension) }\end{array}$ & $\begin{array}{l}4 \text { biases to null: selection } \\
\text { bias likely in assembly of } \\
\text { sample [large percentage } \\
(62 \%) \text { of those undergoing } \\
\text { angiography had no CAD; } \\
\text { may have been selected, at } \\
\text { least in part, because of ex- } \\
\text { posure to untoward job } \\
\text { conditions], survivor bias } \\
\text { as a cross-sectional study, } \\
\text { single cut point for job } \\
\text { strain, low percentage } \\
\text { (10\%) job strain, exclusion } \\
\text { of those with long-standing } \\
\text { angina, or previous MI indi- } \\
\text { cating limitation of range } \\
\text { for outcome (uncertain how } \\
\text { this affects results); total } \\
\text { validity score } 34\end{array}$ \\
\hline \multicolumn{6}{|c|}{ Studies with null results for job strain and cardiovascular disease } \\
\hline $\begin{array}{l}\text { Hall et al, } \\
1993 \text { (35) }\end{array}$ & $\begin{array}{l}\mathrm{N}=5921 \text { women, } \\
\text { Swedish, } 45- \\
74 \text { years of age, } \\
\text { random population } \\
\text { sample }\end{array}$ & $\begin{array}{l}\text { Self-reported } \\
\text { CVD (N= } \\
1147)\end{array}$ & $\begin{array}{l}\text { Work control \& social support } \\
\text { interact in a multiplicative man- } \\
\text { ner with occupational class, } \\
\text { indicating risk greater than that } \\
\text { attributable to class alone }\end{array}$ & $\begin{array}{l}\text { White-collar: job strain }<1 \text {, } \\
\text { psychological demands OR } \\
0.81(95 \% \mathrm{Cl} 0.62-1.06) \text {, low } \\
\text { control OR } 1.23 \text { (95\% Cl } 0.9- \\
\text { 1.69); blue-collar: job strain }<1 \text {, } \\
\text { psychological demand OR } 0.76 \\
\text { (95\% Cl 0.6-0.97), low control } \\
\text { OR } 1.02(95 \% \mathrm{Cl} 0.87-1.2) \\
\text { (age) }\end{array}$ & $\begin{array}{l}4 \text { biases to null: survivor } \\
\text { bias as a cross-sectional } \\
\text { study, imputation method, } \\
2 \text { items for psychological } \\
\text { demands, single cut point } \\
\text { for job strain; information } \\
\text { bias unlikely since only } \\
\text { outcome self-reported; } \\
\text { total validity criteria score } \\
35\end{array}$ \\
\hline $\begin{array}{l}\text { Hlatky et al, } \\
1995 \text { (37) }\end{array}$ & $\begin{array}{l}\mathrm{N}=1132 \text { men, } \\
\mathrm{N}=357 \text { women, } \\
\text { median age } 52 \\
\text { years, US patients } \\
\text { undergoing } \\
\text { coronary angio- } \\
\text { graphy (88\% white, } \\
60 \% \text { white-collar) }\end{array}$ & $\begin{array}{l}\text { Degree of } \\
\text { coronary } \\
\text { atheromatosis }\end{array}$ & & $\begin{array}{l}\text { Job strain: quadrant term RR } \\
0.98 \text { ( } 95 \% \mathrm{Cl} 0.71-1.36) \text {, index } \\
\text { RR } 1.0(95 \% \mathrm{Cl} 0.99-1.01) \\
\text { (age, gender, smoking status, } \\
\text { diabetes hypercholesterolemia, } \\
\text { history of MI, typical angina) }\end{array}$ & $\begin{array}{l}4 \text { biases to null: selection } \\
\text { bias likely in assembly of } \\
\text { sample for those exposed } \\
\text { to job strain and undergo- } \\
\text { ing angiography but with- } \\
\text { out CAD, survivor bias } \\
\text { cross-sectional study, no } \\
\text { gender stratification, con- } \\
\text { founding by SES, job strain } \\
\text { higher among white- } \\
\text { collar workers, but blue- } \\
\text { collar workers had more } \\
\text { CAD; total validity } \\
\text { criteria score } 33\end{array}$ \\
\hline
\end{tabular}

a All available risk estimates with confidence intervals are shown. For an odds or risk ratio to be considered significant, the $95 \% \mathrm{Cl}$ had to exclude 1.0 .

b The relevant confounders that were either matched between groups or were adjusted are italicized and indicated in parentheses.

${ }^{c}$ Issues that could affect the directionality of the results of each study are given.

d Readers who would like to see further details about the methodological issues of a particular study or studies may find it helpful to examine the results

in this table together with the corresponding validity assessments in tables 2-5.

e When gender stratified.

Table 9. Job strain and cardiovascular disease outcomes: summary table. (CHD = coronary heart disease, IHD = ischemic heart disease, $\mathrm{MI}=$ myocardial infarction)

\begin{tabular}{|c|c|c|c|c|c|c|c|}
\hline \multirow[t]{2}{*}{$\begin{array}{l}\text { Job strain } \\
\text { results }\end{array}$} & $\begin{array}{l}\text { Unequivocal bias } \\
\text { to overestimate }\end{array}$ & $\begin{array}{l}\text { Likely bias to } \\
\text { overestimate }\end{array}$ & $\begin{array}{l}\text { Bias possible in } \\
\text { both directions }\end{array}$ & Minimal biases & $\begin{array}{l}\text { Likely bias } \\
\text { to null }\end{array}$ & $\begin{array}{l}\text { Unequivocal bias } \\
\text { to null score }\end{array}$ & $\begin{array}{l}\text { Total } \\
\text { validity } \\
\text { score }\end{array}$ \\
\hline & Study \& score & Study \& score & Study \& score & Study \& score & Study \& score & Study \& Score & Mean SD \\
\hline \multicolumn{8}{|l|}{ Longitudinal studies } \\
\hline \multicolumn{8}{|l|}{ Men } \\
\hline $\begin{array}{l}\text { Significant positive } \\
\text { association }\end{array}$ & - & - & $\begin{array}{l}\text { Theorell et al } \\
(47), \text { score } 35\end{array}$ & $\begin{array}{l}\text { Kuper \& Mar- } \\
\text { mot (42) a, b }\end{array}$ & $\begin{array}{l}\text { Bosma et al (32- } \\
34),{ }^{c} \text { score } 39 \\
\text { Kivimäki et al } \\
(42),{ }^{a} \text { score } 37\end{array}$ & $\begin{array}{l}\text { Alfredsson et al } \\
(30), \text { score } 35 \\
\text { Hammar et al (21, } \\
36) \text {, score } 40 \\
\text { Johnson et al } \\
\text { (38), d score } 37 \\
\text { Karasek et al (40), } \\
\text { score } 36\end{array}$ & 37.31 .9 \\
\hline
\end{tabular}


Table 9. Continued.

\begin{tabular}{|c|c|c|c|c|c|c|c|c|}
\hline \multirow[t]{2}{*}{$\begin{array}{l}\text { Job strain } \\
\text { results }\end{array}$} & \multirow{2}{*}{$\begin{array}{l}\text { Unequivocal bias } \\
\text { to overestimate }\end{array}$} & \multirow{2}{*}{$\begin{array}{l}\text { Likely bias to } \\
\text { overestimate } \\
\text { Study \& score }\end{array}$} & \multirow{2}{*}{$\begin{array}{l}\text { Bias possible in } \\
\text { both directions } \\
\text { Study \& score }\end{array}$} & \multirow{2}{*}{$\begin{array}{l}\text { Minimal biases } \\
\text { Study \& score }\end{array}$} & \multirow{2}{*}{$\begin{array}{l}\text { Likely bias } \\
\text { to null } \\
\text { Study \& score }\end{array}$} & \multirow{2}{*}{$\begin{array}{l}\text { Unequivocal bias } \\
\text { to null score } \\
\text { Study \& Score }\end{array}$} & \multicolumn{2}{|c|}{$\begin{array}{l}\text { Total } \\
\text { validity } \\
\text { score }\end{array}$} \\
\hline & & & & & & & Mean & SD \\
\hline $\begin{array}{l}\text { Nonsignificant } \\
\text { positive association }\end{array}$ & - & - & - & $\begin{array}{l}\text { Kuper \& Mar- } \\
\text { mot }(42)^{a, e}\end{array}$ & $\begin{array}{l}\text { Bosma et al (32- } \\
34),{ }^{f} \text { score } 39\end{array}$ & $\begin{array}{l}\text { Alterman et al } \\
(31), \text { score } 38 \\
\text { Steenland et al } \\
(46), \text { score } 35\end{array}$ & 37.8 & 1.9 \\
\hline Null & - & - & - & - & $\begin{array}{l}\text { Suadicani et al } \\
\text { (23), score } 38\end{array}$ & $\begin{array}{l}\text { Hlatky et al } \\
(37),{ }^{\text {a }} \text { score } 33 \\
\text { Johnson et al } \\
\text { (39), score } 37 \\
\text { Reed et al (45), } \\
\text { score } 40\end{array}$ & 37 & 2.9 \\
\hline $\begin{array}{l}\text { Significant negative } \\
\text { association }\end{array}$ & - & - & - & - & - & - & . & . \\
\hline Total & . & . & . & . & . & . & 37.3 & 2.1 \\
\hline \multicolumn{9}{|l|}{ Women } \\
\hline $\begin{array}{l}\text { Significant positive } \\
\text { association }\end{array}$ & - & - & - & $\begin{array}{l}\text { Kuper \& Mar- } \\
\text { mot }(42)^{a, b}\end{array}$ & $\begin{array}{l}\text { Kivimäki et al } \\
\text { (41), }{ }^{\text {a }} \text { score } 37\end{array}$ & $\begin{array}{l}\text { Alfredsson et al } \\
(30), \text { score } 35 \\
\text { Hammar et al } \\
(21,36) \text {, score } 40\end{array}$ & 37.8 & 2.2 \\
\hline $\begin{array}{l}\text { Nonsignificant } \\
\text { positive association }\end{array}$ & - & - & - & $\begin{array}{l}\text { Kuper \& Marmot } \\
(42)^{a, e}\end{array}$ & $\begin{array}{l}\text { Bosma et al (32- } \\
34),{ }^{9} \text { score } 39 \\
\text { Orth-Gomér et al } \\
(44), \text { score } 37\end{array}$ & - & 38.3 & 1.2 \\
\hline Null & - & - & - & - & - & $\begin{array}{l}\text { Hall et al (35), } \\
\text { score } 35 \\
\text { Hlatky et al (37), a } \\
\text { score } 33 \\
\text { Lee et al (43), } \\
\text { score } 36\end{array}$ & 34.7 & 1.5 \\
\hline $\begin{array}{l}\text { Significant negative } \\
\text { association }\end{array}$ & - & - & - & - & - & - & . & . \\
\hline Total & . & . & . & . & . & . & 37 & 2.3 \\
\hline \multicolumn{9}{|l|}{ Case-control studies } \\
\hline \multicolumn{9}{|l|}{ Men } \\
\hline $\begin{array}{l}\text { Significant positive } \\
\text { association }\end{array}$ & - & - & $\begin{array}{l}\text { Sihm et al (22), } \\
\text { score } 36 \\
\text { Yoshimasu \& the } \\
\text { Fukuoka Heart } \\
\text { Study Group } \\
\text { (60), score } 33\end{array}$ & $\begin{array}{l}\text { Hallqvist et al (54) } \\
\text { \& Theorell et al } \\
(55),{ }^{n} \text { score } 46\end{array}$ & - & $\begin{array}{l}\text { Alfredsson et al } \\
(50,51) \text {, score } \\
38 \text { Netterstrøm et } \\
\text { al }(58) \text {, score } 32 \\
\text { Theorell et al } \\
\text { (59), score } 34\end{array}$ & 36.5 & 5.1 \\
\hline $\begin{array}{l}\text { Nonsignificant } \\
\text { positive association }\end{array}$ & - & - & - & - & $\begin{array}{l}\text { Bobak et al (52), } \\
\text { score } 35\end{array}$ & - & 35 & . \\
\hline Null & & & $\begin{array}{l}\text { Emdad et al (33), } \\
\text { score } 33\end{array}$ & & & & 33 & . \\
\hline $\begin{array}{l}\text { Significant negative } \\
\text { association }\end{array}$ & - & - & - & - & - & - & . & . \\
\hline Total & . & . & . & . & . & . & 35.9 & 4.5 \\
\hline \multicolumn{9}{|l|}{ Women } \\
\hline $\begin{array}{l}\text { Significant positive } \\
\text { association }\end{array}$ & - & - & $\begin{array}{l}\text { Reuterwall et al } \\
(56) \text { \& Peter et al } \\
(57), \text { score } 36\end{array}$ & - & - & - & 36 & . \\
\hline $\begin{array}{l}\text { Nonsignificant } \\
\text { positive association }\end{array}$ & - & $\begin{array}{l}\text { Wamala et al } \\
(60) \text {, score } 38\end{array}$ & - & - & - & - & 38 & . \\
\hline Null & - & - & - & - & - & - & . & . \\
\hline $\begin{array}{l}\text { Significant negative } \\
\text { association }\end{array}$ & - & - & - & - & - & - & . & . \\
\hline Total & . & . & . & . & . & . & 37 & 1.4 \\
\hline \multicolumn{9}{|l|}{ Cross-sectional studies } \\
\hline \multicolumn{9}{|l|}{ Men } \\
\hline $\begin{array}{l}\text { Significant positive } \\
\text { association }\end{array}$ & - & - & $\begin{array}{l}\text { Johnson \& Hall } \\
(5)^{i}, \text { score } 34 \\
\text { Johnson et al } \\
(38),{ }^{d} \text { score } 33 \\
\text { Sacker et al (65), } \\
\text { score } 31\end{array}$ & - & - & $\begin{array}{l}\text { Karasek et al } \\
(63), \text { score } 36\end{array}$ & (contir & nued \\
\hline
\end{tabular}


Table 9. Continued.

\begin{tabular}{|c|c|c|c|c|c|c|c|c|}
\hline \multirow[t]{2}{*}{$\begin{array}{l}\text { Job strain } \\
\text { results }\end{array}$} & \multirow{2}{*}{$\begin{array}{l}\text { Unequivocal bias } \\
\text { to overestimate } \\
\text { Study \& score }\end{array}$} & \multirow{2}{*}{$\begin{array}{l}\text { Likely bias to } \\
\text { overestimate } \\
\text { Study \& score }\end{array}$} & \multirow{2}{*}{$\begin{array}{l}\text { Bias possible in } \\
\text { both directions } \\
\text { Study \& score }\end{array}$} & \multirow{2}{*}{$\begin{array}{l}\text { Minimal biases } \\
\text { Study \& score }\end{array}$} & \multirow{2}{*}{$\begin{array}{l}\text { Likely bias } \\
\text { to null } \\
\text { Study \& score }\end{array}$} & \multirow{2}{*}{$\begin{array}{l}\text { Unequivocal bias } \\
\text { to null } \\
\text { score } \\
\text { Study \& Score }\end{array}$} & \multicolumn{2}{|c|}{$\begin{array}{c}\text { Total } \\
\text { validity } \\
\text { score }\end{array}$} \\
\hline & & & & & & & Mean & SD \\
\hline $\begin{array}{l}\text { Nonsignificant } \\
\text { positive association }\end{array}$ & - & - & $\begin{array}{l}\text { Netterstrøm et al } \\
(64), \text { score } 32\end{array}$ & - & - & $\begin{array}{l}\text { Yoshimasu et al } \\
(66) \text {, score } 34\end{array}$ & 33 & 1.4 \\
\hline Null & - & - & $\begin{array}{l}\text { Johnson }(5),{ }^{j} \\
\text { score } 34\end{array}$ & - & - & $\begin{array}{l}\text { Hlatky et al } \\
(37),{ }^{\text {a }} \text { score } 33\end{array}$ & 33.5 & 0.7 \\
\hline $\begin{array}{l}\text { Significant negative } \\
\text { association }\end{array}$ & - & - & - & - & - & - & . & . \\
\hline Total & . & . & . & . & . & . & 33.4 & 1.5 \\
\hline \multicolumn{9}{|l|}{ Women } \\
\hline $\begin{array}{l}\text { Significant positive } \\
\text { association }\end{array}$ & - & - & - & - & - & - & . & . \\
\hline $\begin{array}{l}\text { Nonsignificant } \\
\text { positive association }\end{array}$ & - & - & $\begin{array}{l}\text { Johnson \& Hall } \\
\text { (5), score } 34\end{array}$ & - & - & - & 34 & . \\
\hline Null & - & - & - & - & - & $\begin{array}{l}\text { Hall et al (35), } \\
\text { score } 35 \\
\text { Hlatky et al } \\
(37),{ }^{\text {a }} \text { score } 33\end{array}$ & 34 & 1.4 \\
\hline $\begin{array}{l}\text { Significant negative } \\
\text { association }\end{array}$ & - & - & - & - & - & - & . & . \\
\hline Total & . & . & . & . & . & . & 34 & 1.0 \\
\hline
\end{tabular}

a Results not gender-stratified.

b All CHD (score 39).

${ }^{c}$ Any CHD end point (self report).

d Results for iso-strain.

e Nonfatal MI or fatal CHD (score 39).

${ }^{\dagger}$ Angina, diagnosed IHD (self-report).

g Self-report.

$\mathrm{h}$ Except for survivor bias as in case-control studies.

i Blue-collar.

White-collar.

dimension since its main source of variance is withinoccupation. This problem may explain the discrepant findings of a significant positive association between job control and CVD but the lack of such discrepancy for psychological demands in several of the longitudinal studies $(31,39,46)$ that relied only on imputation. Another problem with imputation was found in the study by Reed et al (45), the only study in which a significant inverse relation $(\mathrm{P}<0.05)$ was found between job strain and incident coronary heart disease (CHD). This inverse finding was apparent for only one subgroup (acculturated Japanese American men in Hawaii). Exposure status in that study was imputed on the basis of data from the United States as a whole. The authors suggested the possibility "that the actual working conditions to which this cohort was exposed were not accurately represented by this method" [and also] "that the different patterns of results shown by the men divided into Westernized and traditional Japanese groups, indicate that such cultural differences can affect the associations [p 501502]".

In two of the studies with null findings $(23,43)$, the participants had taken part in a previous cohort study, and therefore survivor bias was likely to have been operative in the assembly of the sample. In the research by Lee et al (43) the assessment of these psychosocial job characteristics was performed some 16 years after the initiation of the study, and after which more women in the cohort had actually stopped paid employment than were included in the part of the study concerned with job strain. The likelihood is therefore high that a strong healthy worker effect was operative in the assembly of the sample with respect of the assessment of the effects of job strain on incident CHD. Moreover, $49 \%$ of those exposed to job strain at baseline changed their exposure status during the follow-up period. This change, which undoubtedly attenuated the findings, was not taken into account in the analyses.

Selective attrition from high-strain jobs has been reported to be common among working women generally (48). In respect to a longitudinal study (44) comprised of women who had been hospitalized for an ischemic cardiac event, it is plausible that many of those who had previously been exposed to job strain did not return to work after enduring an episode of CHD. The authors did not provide evidence that would rule out this possibility. Moreover, while the direction in which a likely confounder (marital stress) would affect the results is unclear, the effect of combined exposure to marital stress and job strain was not tested. It is not unreasonable to argue that women falling into that category would 